\title{
The StEllar Counterparts of COmpact high velocity clouds (SECCO) survey
}

\section{Sensitivity of the survey and the atlas of synthetic dwarf galaxies ${ }^{\star}$}

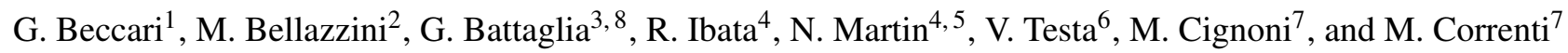 \\ ${ }^{1}$ European Southern Observatory, Karl-Schwarzschild-Strasse 2, 85748 Garching bei Munchen, Germany \\ e-mail: gbeccari@eso.org \\ 2 INAF-Osservatorio Astronomico di Bologna, via Ranzani 1, 40127 Bologna, Italy \\ 3 Instituto de Astrofsica de Canarias, 38205 La Laguna, Tenerife, Spain \\ 4 Observatoire astronomique de Strasbourg, Université de Strasbourg, CNRS, UMR 7550, 11 rue de l'Université, 67000 Strasbourg, \\ France \\ 5 Max-Planck-Institut für Astronomie, Königstuhl 17, 69117 Heidelberg, Germany \\ ${ }^{6}$ INAF-Osservatorio Astronomico di Roma, via Frascati 33, 00040 Monteporzio, Italy \\ 7 Space Telescope Science Institute, 3700 San Martin Drive, Baltimore, MD 21218, USA \\ 8 Universidad de la Laguna, Dpto. Astrofisica, 38206 La Laguna, Tenerife, Spain
}

Received 6 November 2015 / Accepted 10 April 2016

\begin{abstract}
The searching for StEllar Counterparts of COmpact high velocity clouds (SECCO) survey is devoted to the search for stellar counterparts within ultra compact high velocity clouds that are candidate low-mass, low-luminosity galaxies. We present the results of a set of simulations aimed at the quantitative estimate of the sensitivity of the survey as a function of the total luminosity, size, and distance of the stellar systems we are looking for. For all of our synthetic galaxies we assumed an exponential surface brightness profile and an old and metal-poor population. The synthetic galaxies are simulated both on the images and on the photometric catalogues, taking all the observational effects into account. In the fields where the available observational material is of top quality $\left(\simeq 36 \%\right.$ of the SECCO fields), we detect synthetic galaxies as $\geq 5 \sigma$ over-densities of resolved stars down to $\mu_{V, \mathrm{~h}} \simeq 30.0 \mathrm{mag} / \mathrm{arcsec}^{2}$, for $D \leq 1.5 \mathrm{Mpc}$, and down to $\mu_{V, \mathrm{~h}} \simeq 29.5 \mathrm{mag} / \operatorname{arcsec}^{2}$, for $D \leq 2.5 \mathrm{Mpc}$. In the field with the worst observational material of the whole survey, we detect synthetic galaxies with $\mu_{V, \mathrm{~h}} \leq 28.8 \mathrm{mag} / \mathrm{arcsec}^{2}$ out to $D \leq 1.0 \mathrm{Mpc}$, and those with $\mu_{V, \mathrm{~h}} \leq 27.5 \mathrm{mag} / \mathrm{arcsec}^{2}$ out to $D \leq 2.5 \mathrm{Mpc}$. Dwarf galaxies with $M_{V}=-10.0$, with sizes in the range spanned by known dwarfs, are detected by visual inspection of the images up to $D=5 \mathrm{Mpc}$ independent of the image quality. In the best quality images, dwarfs are partially resolved into stars up to $D=3.0 \mathrm{Mpc}$ and completely unresolved at $D=5 \mathrm{Mpc}$. As an independent test of the sensitivity of our images to low surface brightness galaxies, we report on the detection of several dwarf spheroidal galaxies probably located in the Virgo cluster with $M_{V} \lesssim-8.0$ and $\mu_{V, \mathrm{~h}} \lesssim 26.8 \mathrm{mag} / \operatorname{arcsec}^{2}$. The nature of the previously discovered SECCO 1 stellar system, also likely located in the Virgo cluster, is rediscussed in comparison with these dwarfs. While specific for the SECCO survey, our study may also provide general guidelines for the detection of faint stellar systems with $8 \mathrm{~m}$ class telescopes.
\end{abstract}

Key words. galaxies: dwarf - Local Group - galaxies: ISM - galaxies: photometry

\section{Introduction}

Within the standard $\Lambda$-cold dark matter (CDM) cosmology it is generally accepted that baryonic physics has a significant impact in shaping the evolution of dwarf galaxies. All the relevant processes (e.g. reionisation, supernova feedback, and ram-pressure stripping) act by preventing/quenching star formation in lowmass halos $\left(M \leq 10^{9} M_{\odot}\right.$, mini-halos, after Ricotti 2009, and

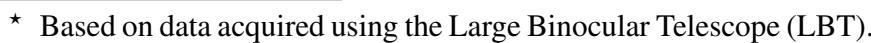
The LBT is an international collaboration among institutions in the United States, Italy, and Germany. The LBT Corporation partners are The University of Arizona on behalf of the Arizona university system; Istituto Nazionale di Astrofisica, Italy; LBT Beteiligungsgesellschaft, Germany, representing the Max-Planck Society, the Astrophysical Institute Potsdam, and Heidelberg University; The Ohio State University; and The Research Corporation, on behalf of The University of Notre Dame, University of Minnesota, and University of Virginia. references therein). Within this framework, the inclusion of baryonic physics in CDM $N$-body models of galaxy formation can strongly mitigate the long standing missing satellites problem (Kauffmann et al. 1993; Klypin et al. 1999; Moore et al. 1999; Kravtsov et al. 2004, and references therein). Independent of the actual ability of these models to capture all the complex events leading to galaxy formation, their general prediction is that there should be a significant number of dark matter mini-halos containing $10^{5}-10^{7} M_{\odot}$ of baryons in the form of neutral hydrogen with or without an associated (small) stellar component (Ricotti 2009; Sawala et al. 2013). The observational detection of these dark and almost-dark galaxies is a crucial frontier for the validation of the current cosmological paradigm on small scales (see e.g. Cannon et al. 2015).

While modern all-sky surveys have allowed us to unveil a large number of very faint gas-deficient galaxies around the 
Milky Way, the so-called ultra faint dwarfs (UFD; Belokurov 2013), these hypothesised gas-rich and star-poor dwarfs have yet to be found. All UFDs were discovered as stellar overdensities; only one of these, Leo $\mathrm{T}$, was subsequently found to have a neutral hydrogen component and ongoing star formation (Irwin et al. 2007). On the other hand, the recent identification of the faint star-forming dwarf galaxy Leo P (Giovanelli et al. 2013) opened a new path for the discovery of these systems. In fact, Leo P was discovered as the stellar counterpart of an ultra compact high velocity cloud (UCHVC) previously identified by the ALFALFA HI survey (Giovanelli et al. 2007). Along this line, Adams et al. (2013, A13 hereafter) selected from the ALFALFA database a sample of 59 UCHVCs lacking any visible counterpart in SDSS images, which they proposed as good candidates to be associated with mini-halos in the distance range $0.25 \mathrm{Mpc} \leq D \leq 2.0 \mathrm{Mpc}$. Similarly, Saul et al. (2013, S12 hereafter) identified 27 UCHVCs from their GALFA-HI survey as local "galaxy candidates" (see Sand et al. 2015, for a critical comparison of the two sources of candidates). The only way to directly confirm these candidates as genuine dwarf galaxies would be to identify a concomitant stellar counterpart whose distance could be estimated, as in the case of Leo P (McQuinn et al. 2013).

The search for stellar counterparts in these UCHVCs is the main scientific goal of the searching for StEllar Counterparts of COmpact high velocity clouds (SECCO) survey ${ }^{1}$ that we presented together with the results from its first phase in Bellazzini et al. (2015a, Paper I hereafter). In particular we used the LBC cameras (Giallongo et al. 2008) mounted at the Large Binocular Telescope ${ }^{2}$ (Mt Graham, AZ) to obtain deep homogeneous wide-field imaging and photometry in two broadbands $(g, r)$ of 25 UCHVCs carefully selected from the A13 sample, plus Leo P, taken as a template. In Paper I we concluded that no obvious stellar counterpart in the range of distances indicated by A13 can be associated with the surveyed UCHVCs. On the other hand, we identified two candidates that are expected to lie at larger distance. The follow-up of one of these candidates revealed that it is indeed a very faint and dark star-forming galaxy likely belonging to the Virgo cluster of galaxies (SECCO 1; Bellazzini et al. 2015b); this result was independently confirmed by Sand et al. (2015).

Several teams worldwide are performing similar surveys (see e.g. Tollerud et al. 2015; Sand et al. 2015; Donovan Meyer et al. 2016). In this context SECCO is the first project, and the only one so far, to perform a systematic homogeneous search, since all the targets were observed with the same set up, albeit not always with the same observing conditions (see Paper I for details and discussion). It is also based on the deepest images; our photometry typically reaches $r \simeq 26.5$. Finally, SECCO was built to allow a quantitative estimate of the significance of nondetections. In Paper I we presented a first limited set of simulations with synthetic galaxies taking all the observational effects into account that lead us to conclude that we did not miss any dwarf with an integrated absolute magnitude $M_{V} \leq-8.0$ and half-light radii $R_{\mathrm{h}} \leq 300 \mathrm{pc}$ lying within $1.5 \mathrm{Mpc}$ from us. In the present contribution we extend our set of experiments with synthetic galaxies to explore the sensitivity limits of our survey in terms of total luminosity, size, and distance of the stellar systems we are looking for. The full range of observational conditions encountered in SECCO is also probed. This is an essential complement to SECCO observations that provides the final complete

\footnotetext{
1 http://www.bo.astro.it/secco/

2 http://www. lbto.org
}

result of our screening at least for the local volume. Moreover, the results of our experiments, performed both on star counts and directly on images, provide a useful set of cases that can be used as a guideline for interpreting the results from similar surveys and to plan new surveys.

The paper is organised as follows: in Sect. 2 we describe the synthetic galaxies we adopt in our study. Section 3 explores the sensitivity of the search for stellar over-densities by means of density maps performed in Paper I. In Sect. 4 we show and discuss our main results on the sensitivity of the visual inspection of images, which are also performed in Paper I. In Sect. 5 we report on the discovery of seven low surface brightness dwarfs likely belonging to the Virgo cluster of galaxy and we use these detections to get an independent validation of the sensitivity of our search by visual inspection. Finally, in Sect. 6 we summarise the main conclusion of our analysis, also briefly rediscussing the results of Paper I in light of the present study. A long table summarising the results of the analysis on density maps is provided in Appendix A, while the images of synthetic galaxies are collected in Appendix B.

\section{Synthetic galaxies}

The synthetic galaxies that we inject into our photometric catalogues, to test the sensitivity of the detection of over-densities in stellar density maps, or in our stacked images, to test the sensitivity of the detection by visual inspection, have the same properties as those adopted and described in Paper I. Here we summarise their main features and illustrate the parameter space that is explored in the present analysis.

The galaxies consist of a synthetic stellar population of old and metal-poor stars obtained from the Bressan et al. (2012) models. The mass of the stars are extracted (down to the hydrogen burning limit) from a mass function $N(m) \propto m^{-x}$ with $x=-2.35$ for $m>0.5 M_{\odot}$ and $x=-1.30$ for $m \leq 0.5 M_{\odot}$. The stars span a small range in metallicity $\left(\sigma_{[\mathrm{Fe} / \mathrm{H}]}=0.1 \mathrm{dex}\right)$ with mean metallicity $[\mathrm{Fe} / \mathrm{H}]=-1.8$ (see Paper I). Star formation starts 13.0 Gyr ago and declines exponentially with time with an exponential scale of 0.5 Gyr. The population is intended to be a fair representation of very faint dwarfs $\left(M_{V} \geq-10.0\right)$ that are known to be dominated by old and metal-poor populations (see e.g. Mateo 1998; McConnachie 2012; Weisz et al. 2014). The choice of a mainly old populations is conservative, since the lack of bright blue stars makes detection more difficult than for a star-forming galaxy. On the other hand the inclusion of a young stellar component in our synthetic galaxies would have implied a strong dependency on further assumptions, for example on star formation efficiency and initial mass function.

The synthetic stars are spatially distributed in the plane of the sky according to an exponential profile ${ }^{3}$. This is known to be an appropriate choice for a wide range of low-luminosity dwarfs (Mateo 1998; Martin et al. 2008) and it is very simple because it is specified only by the value of its half-light radius $R_{\mathrm{h}}$ and a luminosity normalisation (here the integrated magnitude, $M_{V}$, that is fixed by extracting a fraction of the synthetic stellar population that sums up the required total luminosity). Circular (spherical) symmetry is always adopted.

We only consider dwarf galaxies that are fainter than $M_{V}=$ -10.0 . The performed experiments reveal that this choice is adequate, since galaxies with $M_{V} \leq-10.0$ are detected in SECCO

\footnotetext{
3 Here we adopt the formalism introduced by Ciotti \& Bertin (1999) for Sersic models with $R_{\mathrm{h}}=R_{\mathrm{e}}$. The exponential profile is the case $m=1$.
} 
G. Beccari et al.: The StEllar Counterparts of COmpact high velocity clouds (SECCO) survey. II.

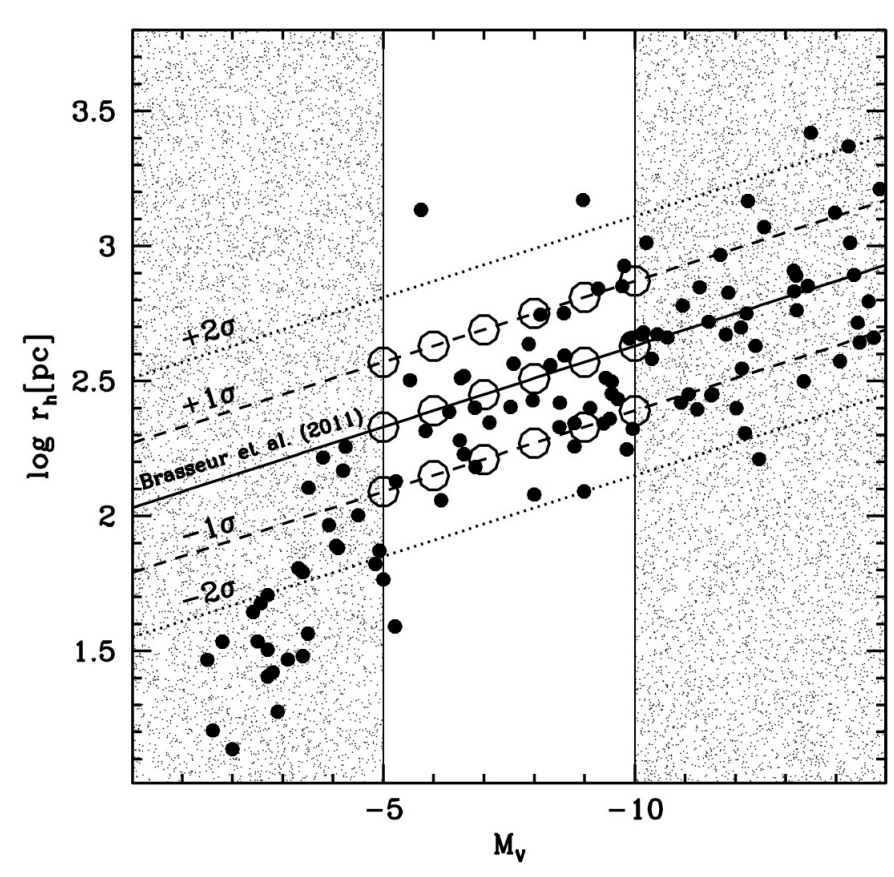

Fig. 1. Integrated absolute $V$ magnitude vs. the logarithm of the halflight radius for dwarf galaxies in the most recent version of the M12 catalogue (filled circles). The mean $M_{V}$ vs. $\log R_{\mathrm{h}}$ relation by Brasseur et al. (2011) is plotted as a continuous line; the curves bracketing $\pm 1 \sigma$ and $\pm 2 \sigma$ range about that relation are plotted as dashed and dotted lines, respectively. The middle, unshaded area of the figure highlights the range of $M_{V}$ considered here. Large empty circles indicate the synthetic dwarf galaxies under study (see also Fig. 2).

over the whole range of parameters relevant for our purposes, independent of the quality of the observational material (see Sect. 4, below). Our results suggest, however, that dwarfs fainter than $M_{V}=-5.0$ would be detected only if they were more compact than average and had $D \lesssim 0.5 \mathrm{Mpc}$. The half-light radius of dwarf galaxies is known to scale with their absolute integrated magnitude (see e.g. Tolstoy et al. 2009; McConnachie 2012, and references therein). While it is likely that this relation is significantly shaped by incompleteness, especially on the low surface brightness side and for $M_{V} \gtrsim-6.0$ (Brasseur et al. 2011), it provides us with a basic guideline for the main goal of the present study, that is to explore the sensitivity of SECCO in the range of known dwarf galaxies. In Sects. 3 and 6 we show that the adopted approach also allows us to probe the sensitivity of the survey in regions of the parameter space that are still poorly explored by existing panoramic surveys, in particular for $\mu_{V, \mathrm{~h}}>26.0 \mathrm{mag} / \operatorname{arcsec}^{2}$ at $D \gtrsim 1.0 \mathrm{Mpc}$.

Brasseur et al. (2011) studied this relation in detail and provided a simple model for the mean half-light radius $\bar{R}_{\mathrm{h}}$ as a function of $M_{V}$ based on Milky Way and M31 satellites with well-measured standard deviations $\sigma_{\log R_{\mathrm{h}}}$ about the mean relation, taking into account the effect of incompleteness

$\log \left(\bar{R}_{\mathrm{h}}\left(M_{V}\right)\right)=2.39+0.24\left(M_{V}+6.0\right)$ with $\sigma_{\log R_{\mathrm{h}}}=0.06$

(see Brasseur et al. 2011, for details). Here, for each value of $M_{V}$, we consider synthetic galaxies with $\log \left(R_{\mathrm{h}}\right)=\log \left(\bar{R}_{\mathrm{h}}\left(M_{V}\right)\right)$, $\bar{R}_{\mathrm{h}}\left(M_{V}\right)-1 \sigma_{\log R_{\mathrm{h}}}$, and $\bar{R}_{\mathrm{h}}\left(M_{v}\right)+1 \sigma_{\log R_{\mathrm{h}}}$ to explore the range of sizes spanned by known dwarfs. In the following we often refer to models with $R_{\mathrm{h}}=\bar{R}_{\mathrm{h}}\left(M_{v}\right), \bar{R}_{\mathrm{h}}\left(M_{v}\right)-1 \sigma_{\log R_{\mathrm{h}}}$, and $\bar{R}_{\mathrm{h}}\left(M_{v}\right)+$ $1 \sigma_{\log R_{\mathrm{h}}}$ as average, compact, and diffuse models, respectively.

In Fig. 1 the Brasseur et al. (2011) model (together with its $\pm 1 \sigma_{\log R_{\mathrm{h}}}$ and $\pm 2 \sigma_{\log R_{\mathrm{h}}}$ envelopes) is compared to the dwarfs in
Table 1. Properties of the synthetic galaxies.

\begin{tabular}{ccc}
\hline \hline$M_{V}$ & $\begin{array}{c}R_{\mathrm{h}} \\
{[\mathrm{pc}]}\end{array}$ & $\begin{array}{c}\mu_{V, \mathrm{~h}} \\
{\left[\mathrm{mag} / \operatorname{arcsec}^{2}\right]}\end{array}$ \\
\hline-10.0 & 245 & 25.5 \\
-10.0 & 426 & 26.7 \\
-10.0 & 741 & 27.9 \\
-9.0 & 213 & 26.2 \\
-9.0 & 371 & 27.4 \\
-9.0 & 645 & 28.6 \\
-8.0 & 186 & 26.9 \\
-8.0 & 323 & 28.1 \\
-8.0 & 562 & 29.3 \\
-7.0 & 162 & 27.6 \\
-7.0 & 281 & 28.8 \\
-7.0 & 489 & 30.0 \\
-6.0 & 141 & 28.3 \\
-6.0 & 245 & 29.5 \\
-6.0 & 426 & 30.7 \\
-5.0 & 123 & 29.0 \\
-5.0 & 213 & 30.2 \\
-5.0 & 371 & 31.4 \\
\hline
\end{tabular}

Notes. All the synthetic galaxies have exponential surface density profiles. $M_{V}$ is the integrated absolute magnitude in $V$ band. $\mu_{V, \mathrm{~h}}$ is the average surface brightness within $R_{\mathrm{h}}$.

the local volume (LV) from the compilation by McConnachie (2012). In the range of $M_{V}$ considered here, systems with $R_{\mathrm{h}} \geq$ $\bar{R}_{\mathrm{h}}\left(M_{v}\right)+1 \sigma_{\log R_{\mathrm{h}}}$ are very rare, while several dwarfs have $R_{\mathrm{h}} \leq$ $\bar{R}_{\mathrm{h}}\left(M_{v}\right)-1 \sigma_{\log R_{\mathrm{h}}}$. This may be partly due to selection effects; however the asymmetry also remains for $M_{V} \leq-8.0$, suggesting that dwarfs that are more compact than average are more frequent than dwarfs that are more extended than average.

The grid of $M_{V}, R_{\mathrm{h}}$, and $\mu_{V, \mathrm{~h}}{ }^{4}$ of the synthetic galaxies considered here is reported in Table 1 and illustrated in Figs. 1 and 2. We only explored knots of the grid if required. For example, if for a given $M_{V}$ the dwarfs with $R_{\mathrm{h}}=\bar{R}_{\mathrm{h}}\left(M_{v}\right)$ are not detected in a given dataset, the exploration of the corresponding $\bar{R}_{\mathrm{h}}\left(M_{v}\right)+1 \sigma_{\log R_{\mathrm{h}}}$ case is pointless, since the detection of more diffuse systems is more difficult.

We adopted a different grid of distances for the density maps and visual inspection experiments. The first are only sensitive to galaxies that are at least partially resolved into stars, hence relatively nearby, but can also reveal very faint and diffuse systems. For this set of experiments we simulate the synthetic dwarfs at distances of $0.25,0.5,1.0,1.5,2.0$, and $2.5 \mathrm{Mpc}$, but visual inspection can also reveal more distant and unresolved systems. In this case we consider the cases $D=0.5,1.0,2.0,3.0$, and $5.0 \mathrm{Mpc}$. A general conclusion from all our experiments with images of synthetic galaxies is that, in agreement with the ruleof-thumb statement made in Paper I, the distance limit at which we partially resolve dwarfs into stars also in the best observational conditions is $D \simeq 3.0 \mathrm{Mpc}$ (see below).

Finally, we performed our experiment using (a) a dataset that is representative of the 36 per cent of the SECCO fields observed under the "best observing conditions" (Field B); and (b) a dataset of the field observed under the "worst observing conditions" of the whole survey (Field I, see Paper I). This is intended to

\footnotetext{
4 Average surface brightness within $R_{\mathrm{h}}$ of the corresponding exponential model, derived as $\mu_{V, \mathrm{~h}}=M_{V}+5 \log R_{\mathrm{h}}+23.567$, where $R_{\mathrm{h}}$ is expressed in parsecs.
} 


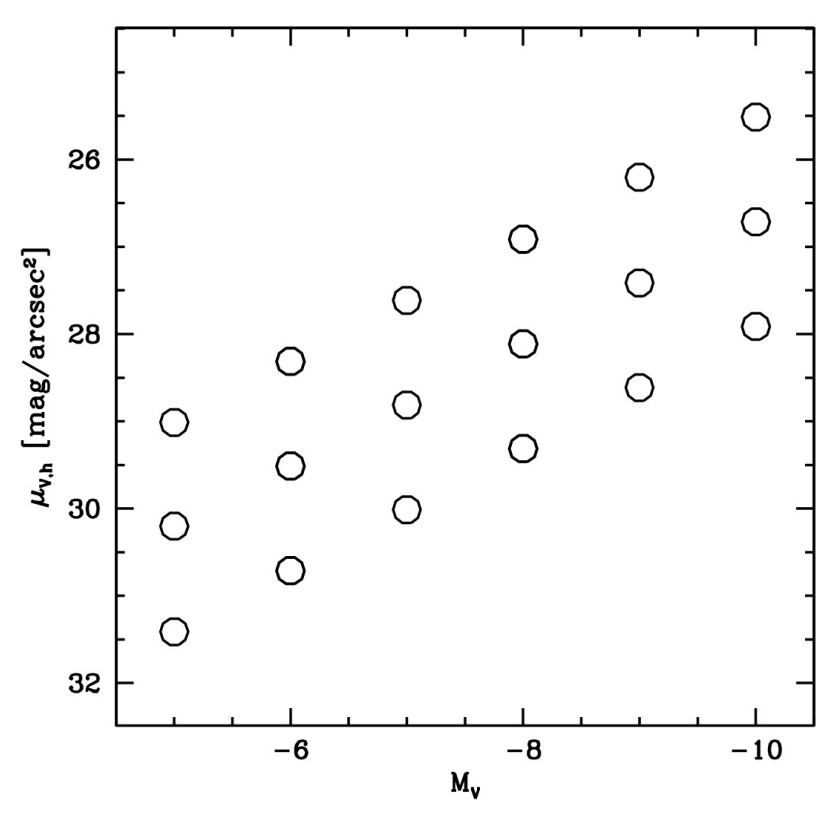

Fig. 2. Integrated absolute $V$ magnitude vs. average surface brightness within $R_{\mathrm{h}}$ for the synthetic dwarf galaxies under study (see also Fig. 1). All of the considered dwarfs have an exponential surface brightness profile.

bracket the whole range of observing conditions encountered in SECCO and to fully assess the impact of the data quality on the sensitivity of the survey. For reference, the images of Field B(I) were obtained with typical seeing of $0.9^{\prime \prime}\left(1.4^{\prime \prime}\right)$ with a limiting magnitude of the final photometry (as traced by the $r_{90}$ parameter, see Paper I) of $r_{90}=26.37\left(r_{90}=25.59\right)$. In the following we often refer to these two cases as the "Best" and "Worst" cases, respectively, for brevity and simplicity.

\subsection{Injecting synthetic galaxies into photometric catalogues}

The process is described in detail in Paper I. To summarise we use the extensive sets of artificial star experiments presented in Paper I to transform the catalogue of synthetic stars for a given $M_{V}$ according to all the effects associated with the observation and data reduction, i.e. incompleteness, photometric errors, and selection effects.

The synthetic catalogue produced in this way is then merged to the original photometric catalogue of the Fields B and I of the SECCO survey and the surface density maps are derived in exactly the same way as in Paper I. Then we annotate whether the synthetic dwarf is detected at $\geq 5 \sigma$ or at $\geq 10 \sigma$ above the background or not detected. We also verify whether the nature of detected over-densities can be firmly established by the inspection of the colour magnitude diagram (CMD) within a given radius (taken as $R_{\mathrm{h}}$, for convenience), compared to the CMD of the surrounding field. This classification process is illustrated in detail in Sect. 3.

\subsection{Injecting synthetic galaxies into stacked images}

The magnitudes and positions of the stars in the synthetic catalogues described in the previous sections are used to inject the synthetic galaxies in the real images with the DAOPHOT/ADDSTAR routine (Stetson 1987). In short, the routine adds simulated stars into the real images in accordance with their positions and magnitudes, using the point spread function (PSF) model characteristic of each image that is used for photometric reduction (see Paper I).

We have a total of four science frames for each field, namely $2 g$ and $2 r$ band exposures. The simulated stars are first added to the best seeing exposure taken as the reference frame. The centre of the synthetic object $\left(X_{0}, Y_{0}\right)$ is placed roughly at the centre of the reference frame and far from saturated stars to avoid contamination from scattered light. It is important to note that heavily saturated stars would prevent the detection of a background dwarf if the size of the circle dominated by the light of the stars on the image is comparable to the size of the dwarf. The synthetic galaxies considered here typically have $r_{\mathrm{h}} \gtrsim 10^{\prime \prime}$ (up to $>60^{\prime \prime}$ ). Identifying bright stars in our images we found that stars with $r \lessgtr 12.0$ are required to reduce significantly the sensitivity over a circle with radius $\gtrsim 15^{\prime \prime}$, stars with $r \lesssim 13.0$ obliterate circles with radius $\gtrsim 10^{\prime \prime}$, and those with with $r \lessgtr 15.0$ obliterate circles with radius $\gtrsim 5^{\prime \prime}$. These bright stars are rare in our high latitude fields ( 19 of 25 have galactic latitude $\left.>50^{\circ}\right)^{5}$. The physical position of each star in the image is simply calculated as $X_{\mathrm{s}}=X_{0}+X_{\mathrm{c}}$ and $Y_{\mathrm{s}}=Y_{0}+Y_{\mathrm{c}}$, where $X_{\mathrm{s}}$ and $Y_{\mathrm{s}}$ are the coordinates with respect to the coordinates in pixel of the centre $X_{\mathrm{c}}, Y_{\mathrm{c}}$.

The four images with the synthetic stars were then fed to MONTAGE2 (Stetson 1987) to build a stacked image for each simulation. These stacked images are used to assess the sensitivity of the SECCO survey through visual inspections (see Sect. 4).

\section{Sensitivity of the density maps}

We consider the case of a $M_{V}=-8.0$ dwarf galaxy to illustrate the process of the detection of synthetic galaxies with density maps. In the upper panel of Fig. 3 we show the maps obtained for a $R_{\mathrm{h}}=\bar{R}_{\mathrm{h}}\left(M_{v}\right)$ model observed in the best conditions and located at the six distance values adopted for this kind of experiments (see Sect. 2.1). It is readily evident that the synthetic galaxy is detected as an over-density at $\geq 10 \sigma$ over the background at all the considered distances. While the angular scale varies significantly with the distance, as obvious, there is no doubt that such a stellar system would have been detected in SECCO.

In the case of $D=0.25 \mathrm{Mpc}$, the over-density exceeds the limits of the studied field ${ }^{6}$ and still emerges clearly above the background. The inspection of the corresponding $\mathrm{CMD}$ in the lower panel of Fig. 3 clearly reveals the presence of an unexpected stellar population, thus confirming the detection beyond any doubt. In the CMDs shown in Fig. 3, and in analogous figures below, stars of the synthetic galaxies and the fore/background population are plotted in grey and black, respectively. The distribution of black points provide a view of the typical CMD of the fore/background population in an adjacent "blank" field of the same area as those shown in the various panels.

The CMD would allow us to unambiguously classify the detected over-densities as local dwarf galaxies, instead of, for example clusters/groups of unresolved galaxies (see Paper I), up to $D=1.0 \mathrm{Mpc}$. In most cases this would also imply that a reliable distance estimate would have been possible with the

\footnotetext{
5 Using the TRILEGAL galactic model (Girardi et al. 2005), we find that the density of $r \lessgtr 13.0$ stars in a field with $(l, b)=\left(90^{\circ}, 45^{\circ}\right)$ is $\simeq 1$ per $40 \mathrm{arcmin}^{2}$. Hence the impact of a chance superposition between a dwarf galaxy and such bright stars should not have a serious impact on our estimate of the sensitivity.

6 That, we recall, is the $17.3^{\prime} \times 7.7^{\prime}$ field covered by the central LBC chip of each SECCO field.
} 

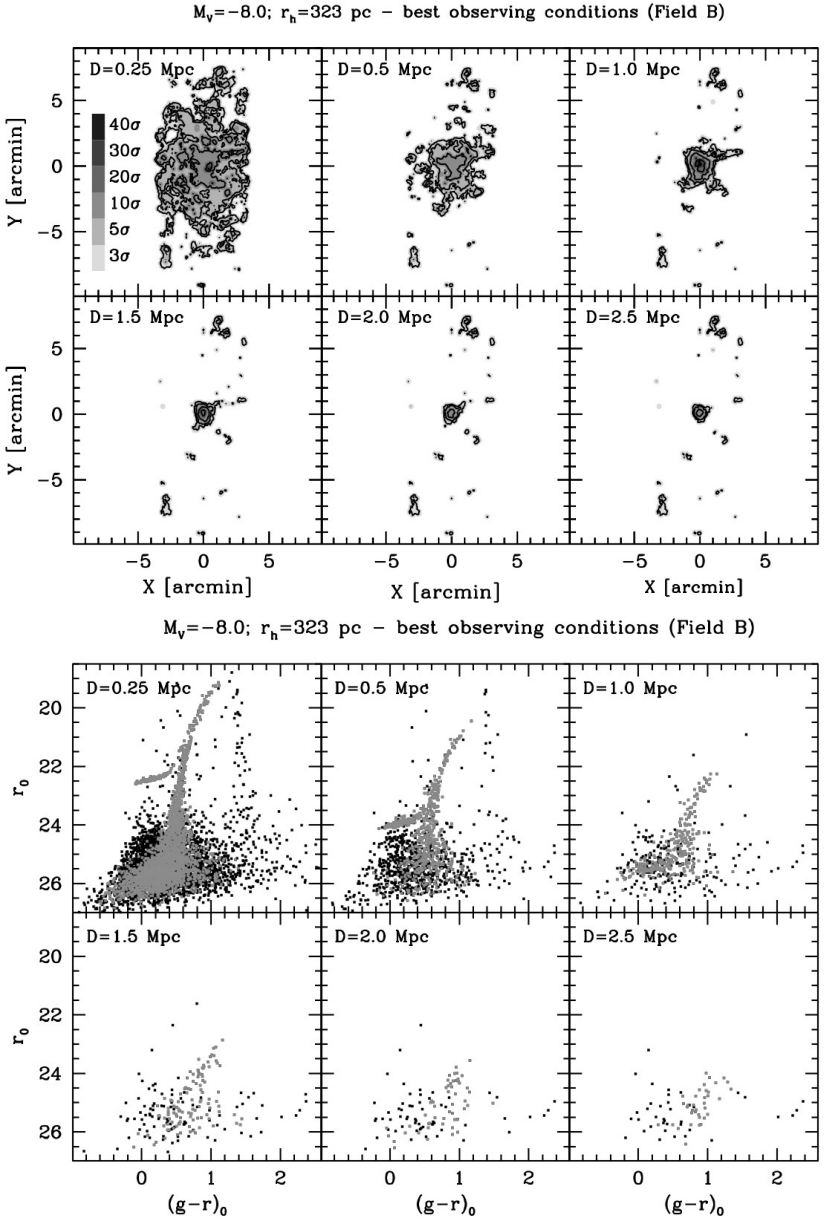

Fig. 3. Upper panel: density maps from a $M_{V}=-8.0$ synthetic galaxy with $R_{\mathrm{h}}=\bar{R}_{\mathrm{h}}\left(M_{v}\right)$ observed in SECCO (Best case). The galaxy is located at six different distances from $D=0.5 \mathrm{Mpc}$ to $D=2.5 \mathrm{Mpc}$. The density scale is in terms of $\sigma$ over the background (see Paper I). Lower panel: CMD of stars within $1 R_{\mathrm{h}}$ of the centre of the synthetic galaxies shown above. Stars from the synthetic population are plotted in grey while field stars are plotted in black.

SECCO observational material, with accuracy depending on the distance, on the total luminosity and stellar population of the considered galaxy. In the case of $D=1.5 \mathrm{Mpc}$, the CMD does not allow an unambiguous classification and reliable distance estimate. However it clearly provides support to the detection, strongly suggesting a follow-up of the candidate. On the other hand, the nature of the clean and compact over-densities detected at $D=2.0 \mathrm{Mpc}$ and $D=2.5 \mathrm{Mpc}$ cannot be established using the CMD, that is too poorly populated and widened by the large photometric errors near the limiting magnitude of the photometry.

Figures 4 and 5 shows the same plots as Fig. 3 but for models with $R_{\mathrm{h}}=\bar{R}_{\mathrm{h}}\left(M_{v}\right)-1 \sigma_{\log R_{\mathrm{h}}}$ and with $R_{\mathrm{h}}=\bar{R}_{\mathrm{h}}\left(M_{v}\right)+1 \sigma_{\log R_{\mathrm{h}}}$, respectively. The comparison of the maps of the compact, average, and diffuse models illustrates the strong impact of the galaxy size on the sensitivity of density maps. As expected, compact galaxies are much more clearly and easily detected. The $\geq 10 \sigma$ over-densities of the $R_{\mathrm{h}}=\bar{R}_{\mathrm{h}}\left(M_{v}\right)$ case become $\geq 40 \sigma$ for the compact model and in some cases reach just $\geq 5 \sigma$ for the diffuse model. By definition, the stars from the synthetic galaxies within $1 R_{\mathrm{h}}$ are the same (for each assumed distance) in all the CMDs for the $M_{V}=-8.0$ galaxy; what changes in the three cases is the actual extension of $R_{\mathrm{h}}$, leading to the inclusion of more and more

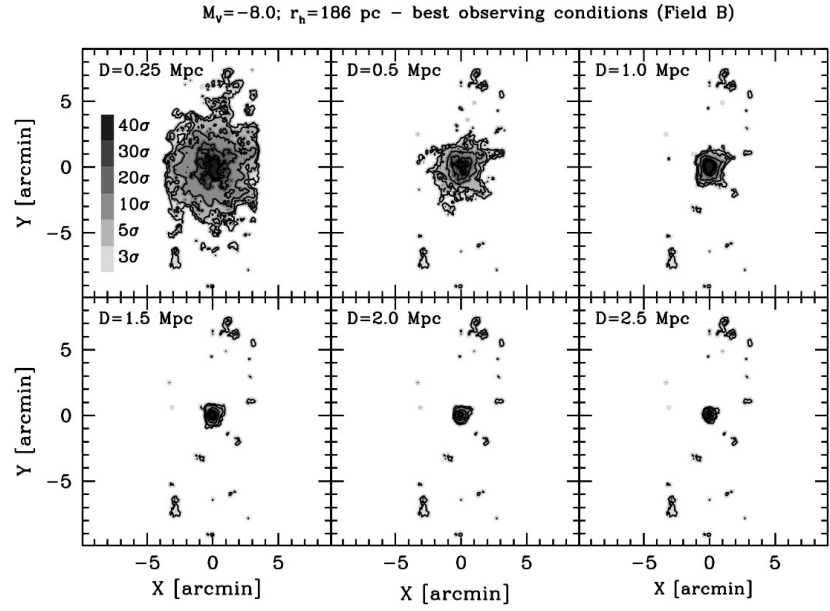

$M_{v}=-8.0 ; r_{h}=186 p c-$ best observing conditions (Field $B$ )

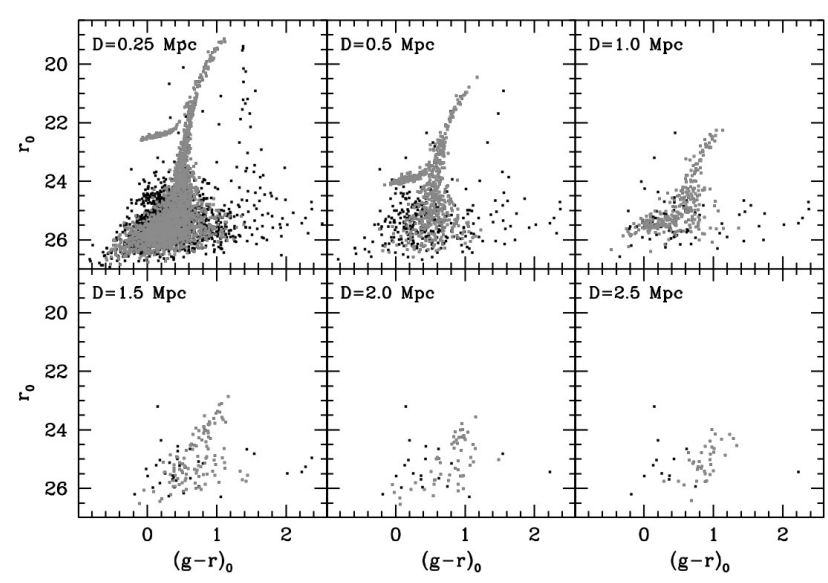

Fig. 4. Same as Fig. 3 for a $M_{V}=-8.0$ synthetic galaxy with $R_{\mathrm{h}}=$ $\bar{R}_{\mathrm{h}}\left(M_{v}\right)-1 \sigma_{\log R_{\mathrm{h}}}$.

field stars in the $R \leq R_{\mathrm{h}} \mathrm{CMD}^{7}$. However, in all of the considered cases the detections for $D \leq 1.5 \mathrm{Mpc}$ are clearly confirmed or strongly supported (for $D=1.5 \mathrm{Mpc}$ ) by the inspection of the CMD.

Figure 6 is strictly analogous to Fig. 3 but for the case of worst quality observational material in SECCO. It can be readily appreciated that, in this case, a $\geq 5 \sigma$ over-density is found only for $D \leq 1.5 \mathrm{Mpc}$, while more distant galaxies would not be detected. However, in spite of the relatively weak over-density signal, the CMD would provide fairly clear confirmations up to $D \leq 1.0 \mathrm{Mpc}$ and, possibly, also to $D \leq 1.5 \mathrm{Mpc}$.

Finally, Fig. 7 shows that, in the worst observing conditions, a $M_{V}=-8.0$ dwarf with $R_{\mathrm{h}}=\bar{R}_{\mathrm{h}}\left(M_{v}\right)+1 \sigma_{\log R_{\mathrm{h}}}$ would never be detected as an over-density in SECCO. In some cases their presence could be revealed by the inspection of the overall CMD of the associated field (in particular for $D \leq 0.5 \mathrm{Mpc}$; see e.g. lower panel of Fig. $6, D=0.25 \mathrm{Mpc}$ case). However, in the present study we consider only significant $(\geq 5 \sigma)$ over-densities as valid detections to avoid ambiguity in our definition of sensitivity and

7 We verified that in our models the angular half-light radius is a good approximation of the radius enclosing the $3 \sigma$ iso-density contour for detected over-densities. Hence, the adoption of $R \leq R_{\mathrm{h}}$ for the CMDs shown here is just a convenient, simple, and uniform choice that should not seriously bias our ability of confirming/detecting a galaxy from its CMD. In Paper I we checked the CMDs over a wide range of radii for all of the $\geq 5 \sigma$ over-densities detected in our density maps. 

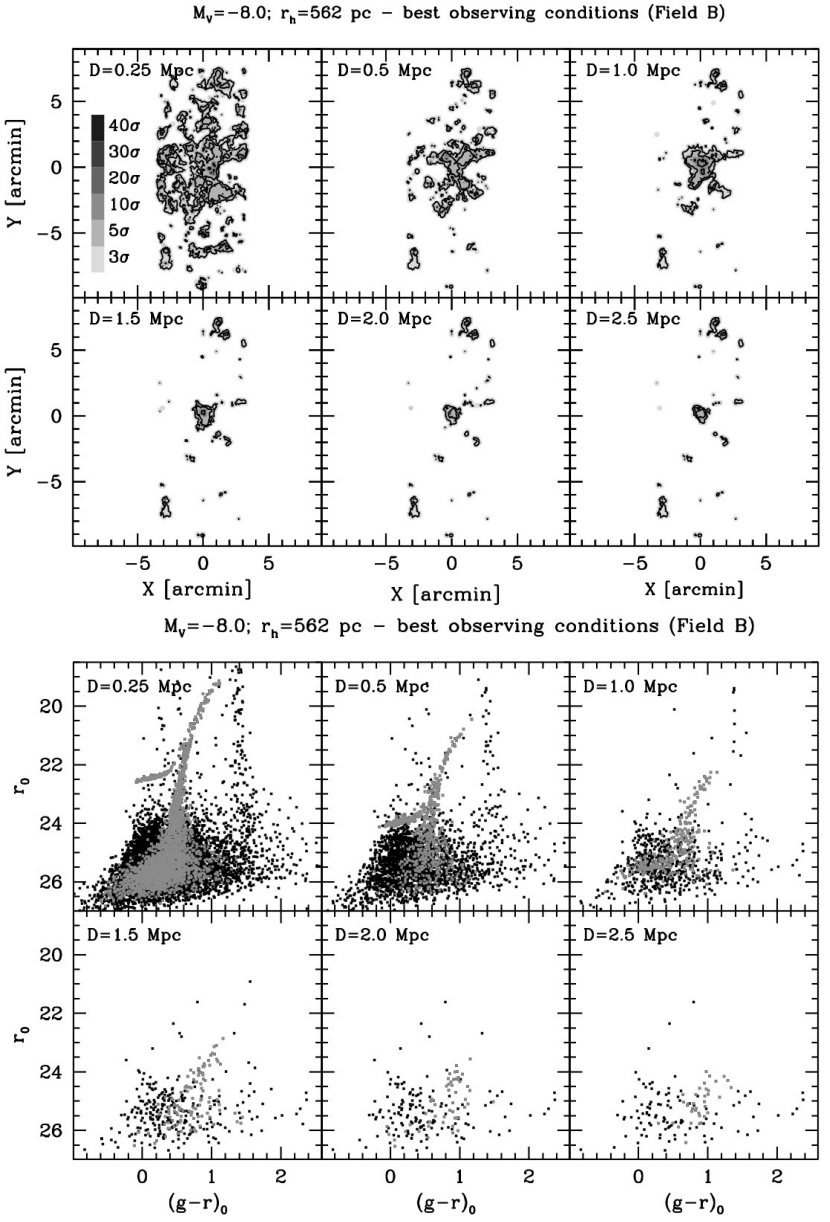

Fig. 5. Same as Fig. 3 for a $M_{V}=-8.0$ synthetic galaxy with $R_{\mathrm{h}}=$ $\bar{R}_{\mathrm{h}}\left(M_{v}\right)+1 \sigma_{\log R_{\mathrm{h}}}$.

only use the CMDs to support the classification of detected objects as genuine dwarfs.

The analysis described above for the $M_{V}=-8.0$ case has been repeated for all the relevant knots of the grid in Table 1.

A graphical representation of the main results of this analysis is shown in Figs. 8 and 9. For any considered case and any adopted distance, we classify the synthetic galaxies and symbolise them in the figures according to the following criteria:

1. the galaxy is detected as an over-density at $\geq 10 \sigma$ (dark grey solid square);

2. the galaxy is detected as an over-density at $\geq 5 \sigma$ but $<10 \sigma$ (light grey solid square);

3. the galaxy is not detected at all (not represented).

Moreover we over-plot a black open square on top of the symbols that represent the cases in which the classification as dwarf galaxy is significantly supported by their CMD. The results of this analysis are shown in Figs. 8 and 9 for Best and Worst case observations, respectively, and are summarised in tabular form in Table A.1. Figure 8 shows that any galaxy with $M_{V} \leq-8.0$ and $D \leq 2.5 \mathrm{Mpc}$ would have been detected by SECCO observations independent of its actual size, in the $\bar{R}_{\mathrm{h}}\left(M_{v}\right) \pm 1 \sigma_{\log R_{\mathrm{h}}}$ range considered here, if it fell within one of the best-quality SECCO fields. In the Best case observations, galaxies with $M_{V} \leq-7.0$ are detected out to $D=2.0 \mathrm{Mpc}$ if they have $R_{\mathrm{h}} \leq \bar{R}_{\mathrm{h}}\left(M_{v}\right)$ and out to $D=1.5 \mathrm{Mpc}$ if they are diffuse. In the case of compact galaxies, systems as faint as $M_{V}=-5.0$ can also be detected
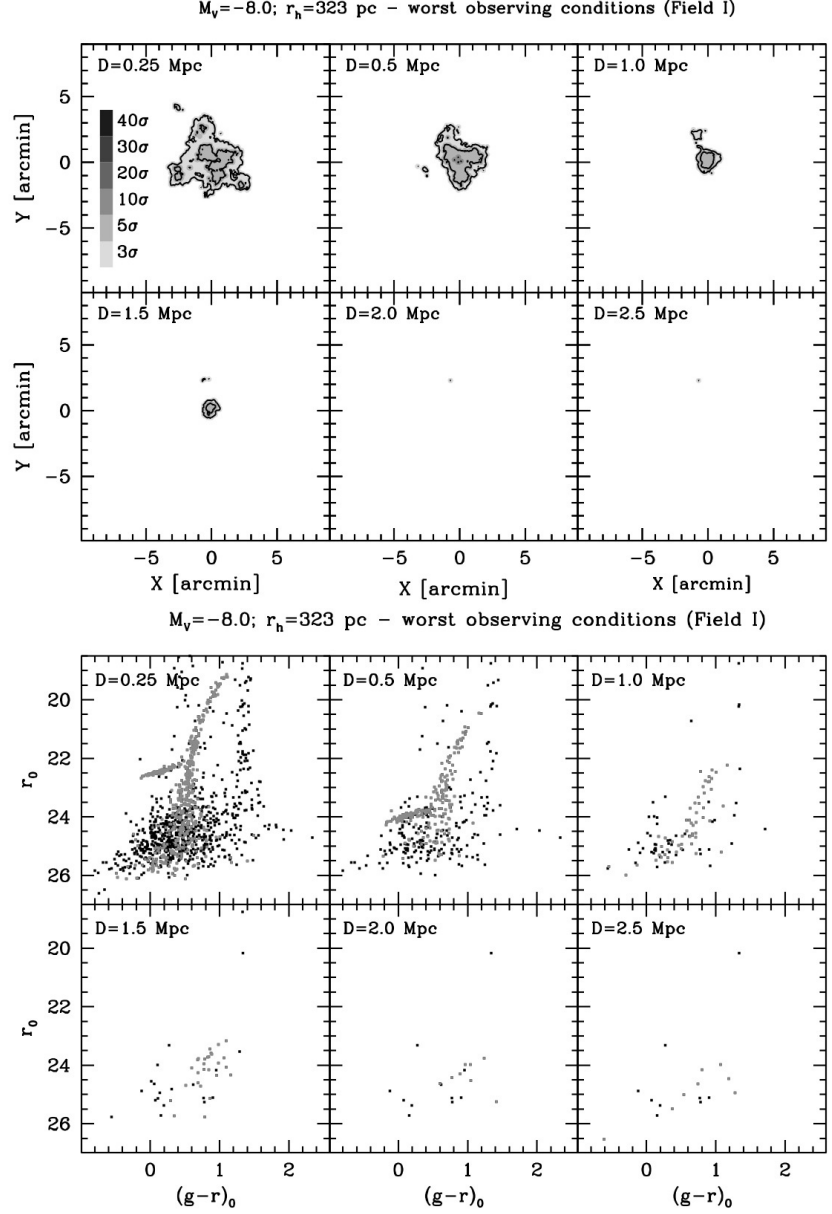

Fig. 6. Same as Fig. $3\left(R_{\mathrm{h}}=\bar{R}_{\mathrm{h}}\left(M_{v}\right)\right.$ models) but for observations in SECCO ("Worst case").

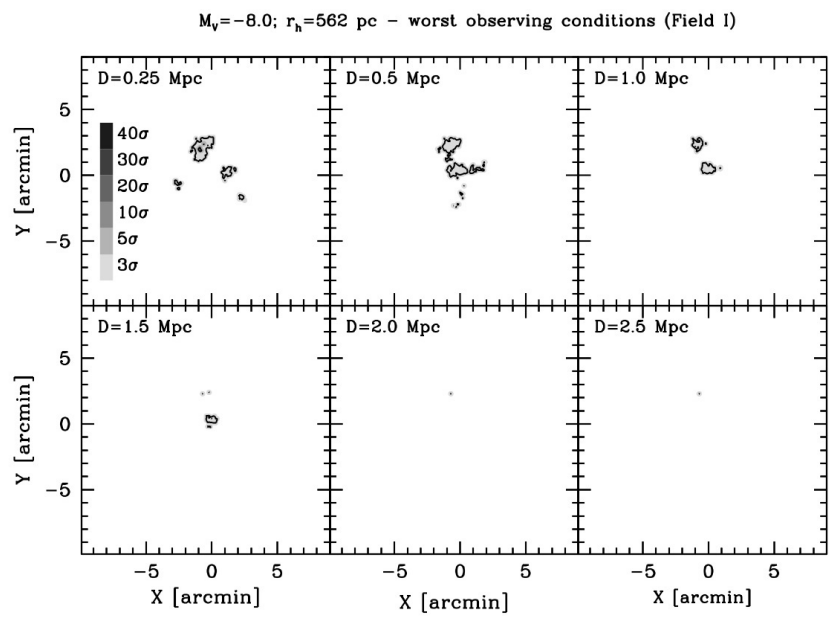

Fig. 7. Density maps for the same case as Fig. 6 but for a diffuse model.

out to $D=1.0 \mathrm{Mpc}$. In all of the panels of this figure we overplotted the distribution of dwarf galaxies in the LV in the considered range of $M_{V}$, from the catalogue by McConnachie (2012) for reference. We note that, in the considered case, any known dwarf with $M_{V} \leq-6.0$ would have been detected in SECCO. Moreover, the sensitivity of our survey extends to much larger distances than those spanned by known dwarfs in the range $-7.0 \leq M_{V} \leq-9.0$ (see Sect. 6 for further discussion on this point). 


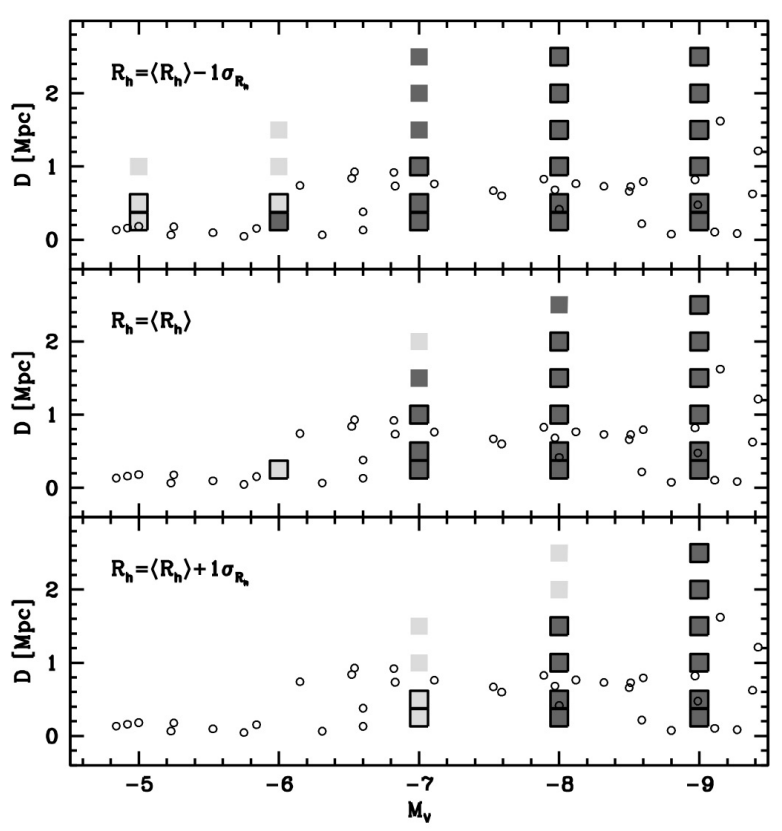

Fig. 8. Summary of density map sensitivity experiments for "Best case" SECCO observations. Light-grey squares indicate $\geq 5 \sigma$ but $<10 \sigma$ overdensity detections, and dark-grey squares correspond to $\geq 10 \sigma$ overdensity detections; the filled squares outlined in black correspond to cases where the CMD provides significant support to the detection. Light open circles are dwarf galaxies in the LV in the considered range of $M_{V}$ from the catalogue by McConnachie (2012). The three panels correspond to compact (upper panel), average (middle panel), and diffuse (lower panel) galaxy models.

In the worst-quality data (Fig. 9), we can still detect any dwarf with $R_{\mathrm{h}} \leq \bar{R}_{\mathrm{h}}\left(M_{v}\right)$ out to $D=2.0 \mathrm{Mpc}$ for $M_{V}=-9.0$ and out to $D=1.5 \mathrm{Mpc}$ for $M_{V}=-8.0$. Compact dwarfs are seen out to $D=1.0 \mathrm{Mpc}$ for $M_{V}=-7$ as well, while only the brightest and most nearby diffuse galaxies can be detected. We show below that for $M_{V} \leq-10.0$ even diffuse galaxies would have been detected by visual inspection in SECCO images, independent of the considered dataset.

In the majority of the considered cases, the nature of the detected (or undetected) over-density can be ascertained from the inspection of its CMD. Finally, within the considered grid of models, the lowest central surface brightness corresponding to $\mathrm{a} \geq 5 \sigma$ over-density is $\mu_{V, \mathrm{~h}}=30.0 \mathrm{mag} / \operatorname{arcsec}^{2}$.

\section{Sensitivity of the visual inspections}

In this section we provide a basic exploration of the process of the search of stellar counterparts by visual inspection of the images. This is mainly intended (a) to assess the luminosity limit above which a typical dwarf cannot be missed on our image; (b) to provide general guidelines on what we can expect to detect in our images; and (c) to provide a framework to interpret actual detections, obtaining rough constrains on the characteristic of a detected system by comparison with images of synthetic galaxies.

In Fig. 10 we show few examples of portions of "Worst case" images centred on a synthetic dwarf galaxy that was added as described in Sect. 2.2. These images provide an idea of the quality of the adopted datasets and of the realism of the simulations. A set of figures analogous to Fig. 10 are presented in Appendix B

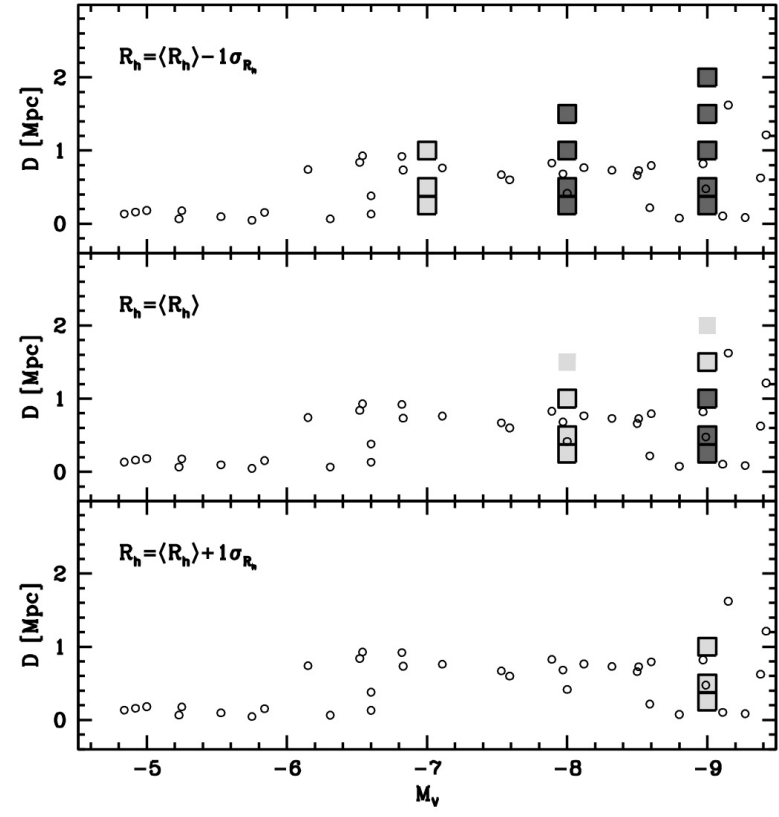

Fig. 9. Summary of the density map sensitivity experiments for "Worst case" SECCO observations. The meaning of the symbols is the same as in Fig. 8.

for the most relevant knots of the grid of considered models (Table 1).

The first three panels of Fig. 10 (from left to right and from top to bottom) show images of a synthetic dwarf galaxy with $M_{V}=-10.0$ and $R_{\mathrm{h}}=\bar{R}_{\mathrm{h}}\left(M_{v}\right)$ located at distances of 1,2 , and $5 \mathrm{Mpc}$, respectively. The fourth (lower right corner) shows the original "empty" image, for reference. A circle of radius equal to the half-light radius is over-plotted, for reference and its value in arcsec is also reported.

In the case of the visual inspection, the examination of the stacked images including synthetic dwarfs allowed us to draw the following conclusions:

1. The $M_{V} \leq-10.0$ dwarfs out to $D=5 \mathrm{Mpc}$ would have been detected in SECCO by visual inspection, independent of the quality of the available observational material and of the galaxy size, within $R_{\mathrm{h}}=\bar{R}_{\mathrm{h}}\left(M_{v}\right) \pm 1 \sigma_{\log R_{\mathrm{h}}}$. As unassuming as it may appear (Fig. B.1), even the diffuse models are clearly noticed when the images are carefully inspected as we did for the real SECCO images (Paper I; see Sect. 5, for real examples). These very low surface brightness galaxies are pretty evident when a simple Gaussian smoothing of a few pixels is applied to the images.

2. The effect of galaxy size is even stronger in images than in density maps. In fact, it turns out that no diffuse model is detected by visual inspection for $M_{V} \geq-9.0$; following this section we do not deal with diffuse models anymore.

3. In our survey, galaxies can be partially resolved up to $D \simeq$ 3.0 Mpc. As unresolved systems they can be detected out to relatively large distances (see, again, Sect. 5) .

The results of the critical analysis of the entire set of images with synthetic galaxies is summarised in Fig. 11. It is clear that visual inspection is a useful complement to the density map analysis, but does not significantly enhance the sensitivity of the survey in the range of distances that is of primary interest for SECCO, i.e. $0.25 \mathrm{Mpc} \leq D \leq 2.0 \mathrm{Mpc}$ (see Paper I). 


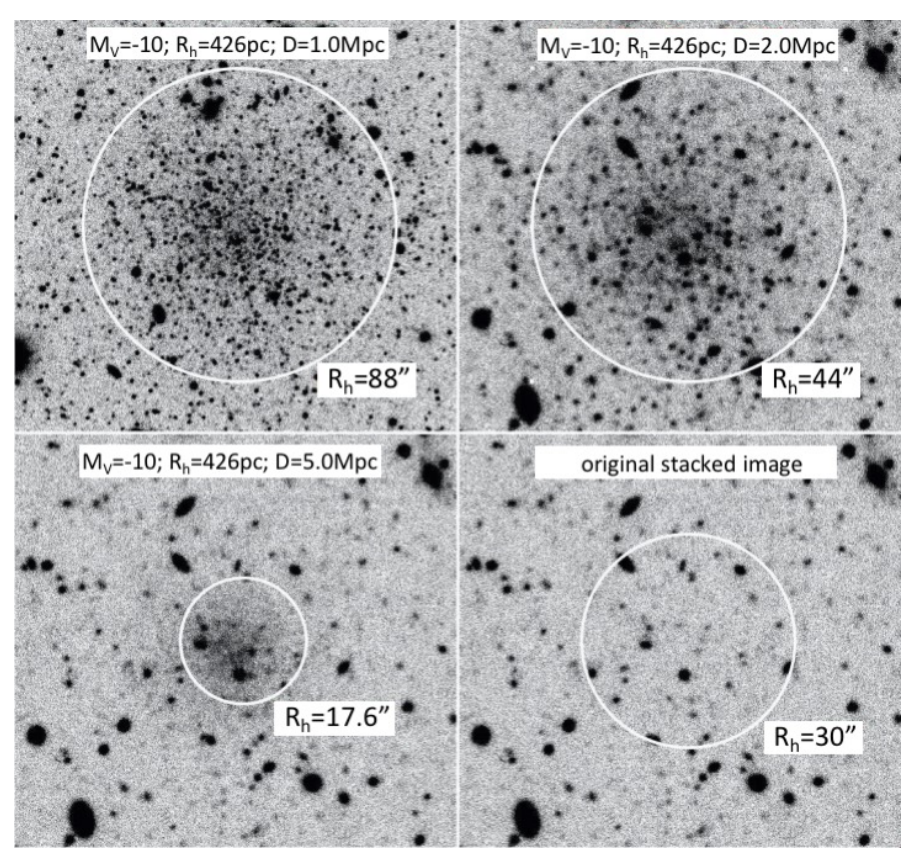

Fig. 10. Synthetic dwarf galaxies of $M_{V}=-10.0$ added to SECCO "Worst case" images for different assumptions of the distance. All the stamp images are centred on the centre of the synthetic galaxy; a circle with radius $=R_{\mathrm{h}}$ is over-plotted. The stamp image in the lower right corner is the original image, without a synthetic galaxy, that is shown for reference with a conventional circle of radius $=30^{\prime \prime}$.

On the other hand, visual inspection extends the sensitivity of the survey to larger distances, especially for compact models. Once again, the example of SECCO 1 (at $D \simeq 16.5 \mathrm{Mpc}$ ) is fairly illustrative: the curious configuration of a few blue stars triggered the attention during the visual inspection of the images but was largely below the detection threshold in density maps (Paper I). In Sect. 5 we empirically demonstrate our ability to spot unassuming spheroidal dwarfs as faint as $M_{V} \sim-8.0$ at the distance of the Virgo cluster, independent of their position in the field.

The synthetic galaxy with the lowest surface brightness that we detect by visual inspection has $\mu_{V, \mathrm{~h}}=28.1 \mathrm{mag} / \mathrm{arcsec}^{2}$, almost a magnitude of two brighter than the limit achieved with density maps. This can be taken as the fundamental sensitivity limit for visual inspection of SECCO images ${ }^{8}$.

We are aware that the simple approach adopted for this part of the analysis may suffer from significant bias in favour of detection, especially for faint dwarfs $\left(M_{V}<-9.0\right)$. A bias may be caused, for example, by placing the synthetic galaxy near the centre of the field and by avoiding the superposition with heavily saturated stars (see Sect. 2.2). We should have produced a large number of images in which synthetic galaxies were added at random position within the field and/or not added at all to fully reproduce the actual process of visual inspection performed on real data; then two of us should subsequently have independently inspected these images, as done in Paper I. However, such an expensive approach would not add any significant piece of information with respect to the main goal of the overall analysis, since, as mentioned above, density maps have a much higher

\footnotetext{
8 With a simple rescaling on aperture photometry we obtained rough estimates of the magnitude and surface brightness of the faintest among the Virgo dwarf spheroidal presented in Sect. 5, VC1 and VC2. We find $M_{V} \sim-7.0$ and $\mu_{V, \mathrm{H}} \sim 27.8 \mathrm{mag} / \operatorname{arcsec}^{2}$ (VC1), which is fully consistent with the limits from synthetic dwarfs.
}
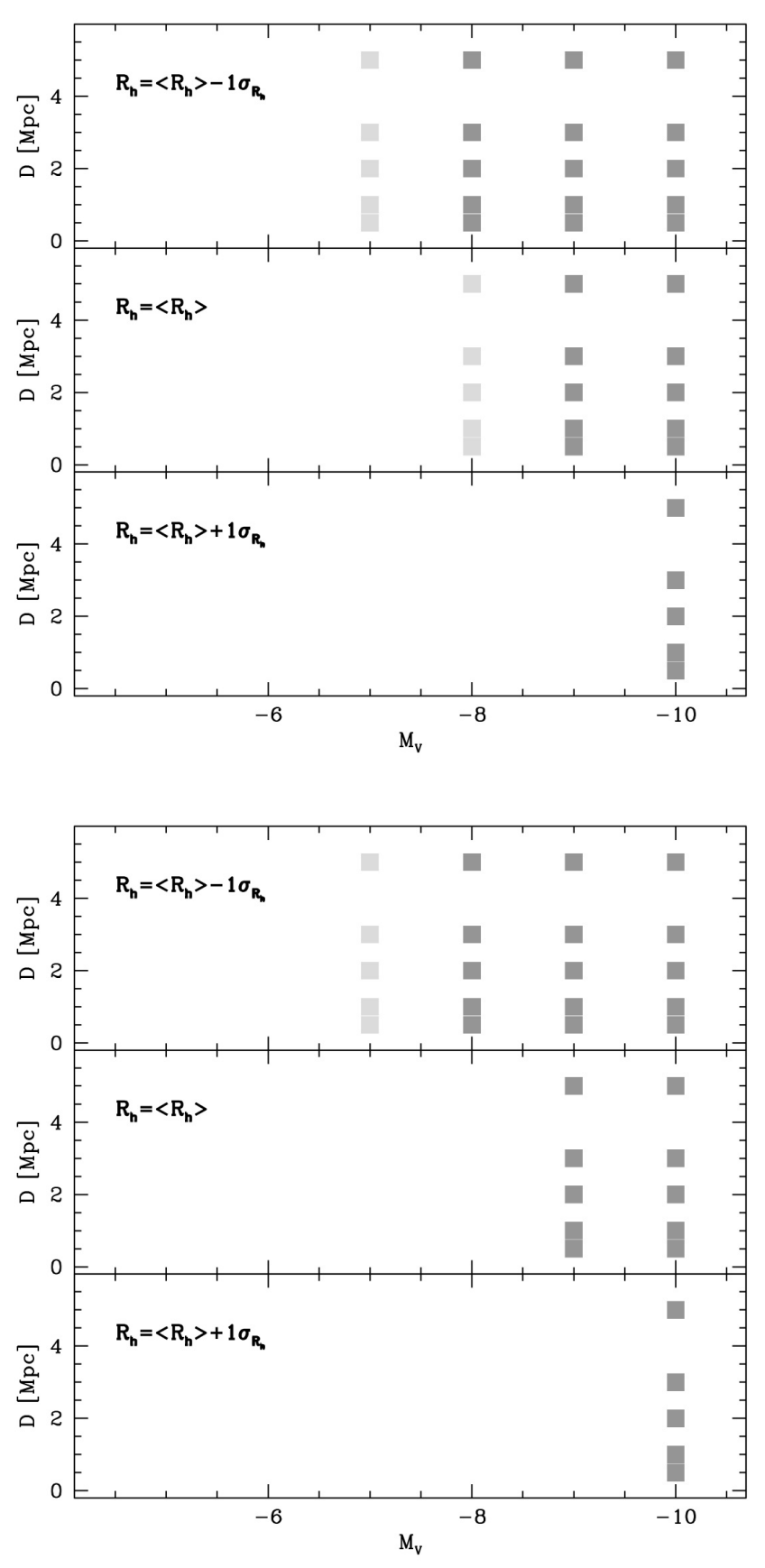

Fig. 11. Summary of the visual inspection sensitivity experiments for "Best case" (upper panels) and "Worst case" images. Dark and light grey squares indicate a clear and doubtful detection, respectively.

sensitivity than visual inspection in the crucial region of the parameter space. The basic procedure described in this section, however, is adequate to get a quantitative idea of the visibility of dwarfs at larger distances than those sampled by the density maps. Finally, in Fig. 11 we distinguish between cases in which the galaxy cannot be missed and less obvious detections, thus providing additional guidance on this aspect.

\section{An empirical test: low surface brightness dwarfs in Virgo}

During the visual inspection of SECCO images (Paper I) we identified seven fluffy and roundish unresolved low surface 
G. Beccari et al.: The StEllar Counterparts of COmpact high velocity clouds (SECCO) survey. II.

Table 2. Candidate low surface rightness dwarfs in the Virgo cluster.

\begin{tabular}{lccccccc}
\hline \hline ID & $\begin{array}{c}\mathrm{RA}_{J 2000} \\
{[\mathrm{deg}]}\end{array}$ & $\begin{array}{c}\text { Dec }_{J 2000} \\
{[\mathrm{deg}]}\end{array}$ & $\begin{array}{c}g \\
{[\mathrm{mag}]}\end{array}$ & $\begin{array}{c}r \\
{[\mathrm{mag}]}\end{array}$ & $\begin{array}{c}R_{\mathrm{h}} \\
{[\mathrm{arcsec}]}\end{array}$ & $\begin{array}{c}\mu_{V, \mathrm{~h}} \\
{\left[\mathrm{mag} / \mathrm{arcsec}^{2}\right]}\end{array}$ & $\begin{array}{c}D_{M 87} \\
{[\mathrm{deg}]}\end{array}$ \\
\hline SECCO-LSB-VC1 & 182.26566 & +4.62742 & & & & & 9.4 \\
SECCO-LSB-VC2 & 182.26848 & +4.61088 & & & & & 9.4 \\
SECCO-LSB-VD1 & 185.28652 & +13.59057 & $\sim 19.5$ & $\sim 18.1$ & $\sim 13.2$ & $\sim 26.3$ & 2.6 \\
SECCO-LSB-VD2 & 185.28589 & +13.57372 & $\sim 23.2^{a}$ & $\sim 22.6^{a}$ & $\sim 2.5^{a}$ & $\sim 26.8^{a}$ & 2.6 \\
SECCO-LSB-VD3 $^{185.34763}$ & +13.58349 & $\sim 20.6$ & $\sim 19.8$ & $\sim 5.8$ & $\sim 25.9$ & 2.6 \\
SECCO-LSB-U1 $^{c}$ & 167.39125 & +5.31381 & & & & & 2.3 \\
SECCO-LSB-VX1 $^{188.48816}$ & +8.40119 & & & & & 4.1 \\
\hline
\end{tabular}

Notes. The last group of letters in the ID (after SECCO-LSB-, whose meaning is obvious) may contain a $V$, indicating the likely association with Virgo, the letter of the SECCO field where the galaxy has been found (in this case Field C, Field D and Field X; see Paper I), anda number to distinguish between dwarfs lying in the same SECCO field. $D_{\mathrm{M} 87}$ is the angular distance from the M 87 galaxy, taken as a proxy for the centre of the Virgo cluster. Integrated magnitudes and surface brightness values were corrected for reddening assuming $E(B-V)=0.048$ (Paper I). (a) Particularly uncertain because of the faintness of the object. ${ }^{(b)}$ Same coordinates, within 1.0", of LSBVCC357 listed by Davies et al. (2016). (c) This galaxy lies $\sim 4^{\circ}$ beyond the zero velocity boundary of Virgo as estimated by Karachentsev \& Nosonova (2010), between the Leo I and Leo II galaxy groups, both lying at a distance of about 10-12 Mpc from us, according to the NED database.

brightness (LSB) galaxies with typical diameters of $20^{\prime \prime}-40^{\prime \prime}$. Six of them lie in three of the four (over 25) SECCO fields that are projected within the wide boundaries of the Virgo cluster of galaxies (Kim et al. 2014). The Virgo cluster is known to host a large population of faint LSB galaxies that can be classified as dwarf spheroidals (dSphs; see e.g. Phillips et al. 1998; Sabatini et al. 2003; Caldwell 2006, and references therein). It is likely that a large portion of this population is still to be discovered; the ongoing Next Generation Virgo Survey (NGVS; Ferrarese et al. 2012) is expected to provide a fundamental contribution in this respect (see e.g. Davies et al. 2016; Mihos et al. 2015). Hence it is reasonable to assume that the LSB galaxies we discovered in SECCO are physically associated with the Virgo cluster.

We briefly report on the LSB galaxies for two main reasons. First they are interesting on their own, since they appear among the faintest and lowest surface brightness dwarfs ever discovered in Virgo, also showing intriguing clustering properties. Second, and most relevant for the present paper, they provide real examples of how faint LSB dwarfs have actually been found by visual inspection in SECCO, thus giving independent support to our sensitivity analysis based on synthetic galaxies. Finally, three of these galaxies have been found in the same images where SECCO 1 (also likely lying in Virgo) has been found (Field D, see Paper I and Bellazzini et al. 2015b) thus providing the basis for a fruitful comparison with that stellar system, which appears peculiar in various aspects. The seven LSB systems are listed in Table 2, where the adopted naming convention is also defined. In the following we drop the "SECCO-LSB" suffix for brevity. None of the listed systems is found in the $\operatorname{Simbad}^{9}$ or $\mathrm{NED}^{10}$ astronomical databases, nor in the recent catalogues by Kim et al. (2014) and Davies et al. (2016, with one exception, see Table 2) ${ }^{11}$.

Figure 12 shows the three LSB dwarfs found in Field D in a single zoomed image. This is possible because they are remarkably close together. Of the six candidate dSphs that we find in the $2131 \operatorname{arcmin}^{2}$ covered by the four SECCO fields sampling

\footnotetext{
9 http://simbad.u-strasbg.fr/simbad/

${ }^{10}$ http://ned.ipac. caltech. edu

${ }^{11}$ Some of these systems are clearly also seen in NGVS images when inspected through the web interface www4.cadc-ccda.hia-iha. nrc-cnrc.gc.ca/en/megapipe/access/graph.html
}

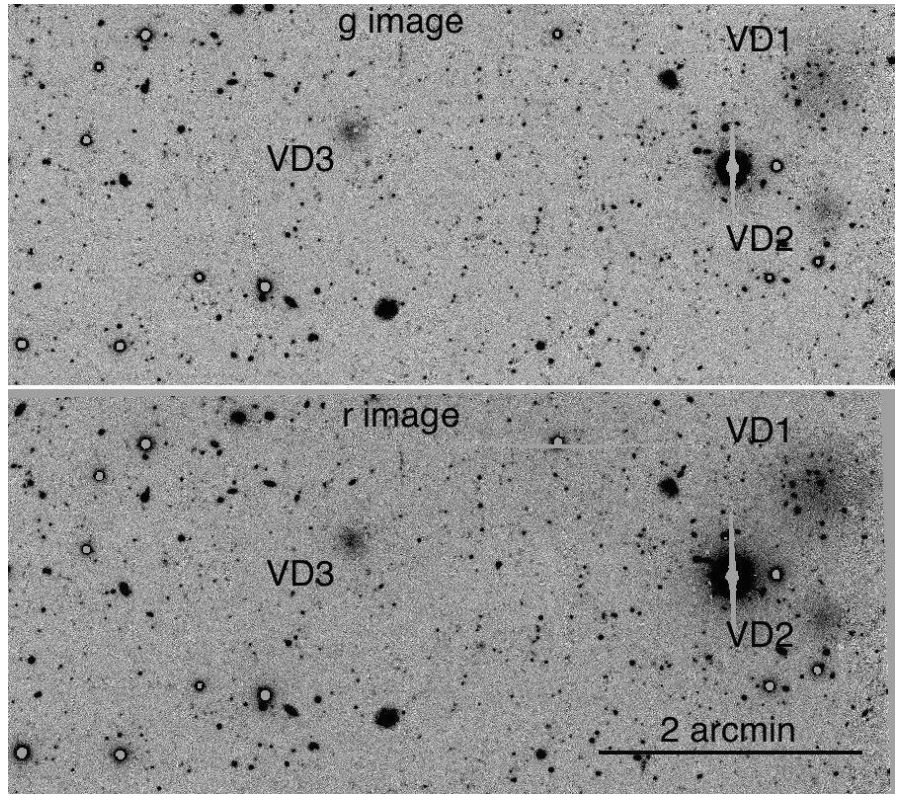

Fig. 12. A portion of Field D $g$-band (upper panel) and $r$-band (lower panel) images enclosing the three LSB galaxies identified in this field. North is up, east to the left.

Virgo, (these) three lie within a circle of area $\simeq 11 \operatorname{arcmin}^{2}$, which is hardly consistent with a random distribution, thus suggesting a physical association (see below for the case of $\mathrm{VC1}$ and VC2). At the distance of $16.5 \mathrm{Mpc}$ (adopted as the mean distance to Virgo; Mei et al. 2007), the projected separation between the centres of VD1 and VD2 is $4.8 \mathrm{kpc}$, a distance comparable with the sum of their apparent diameters; VD3 is just $17.5 \mathrm{kpc}$ from VD1. While inspecting NGVS images we noted another similar LSB dwarf lying just beyond the northern limit of the SECCO image of Field $D$, at $\simeq 5.2^{\prime} \simeq 25.0 \mathrm{kpc}$ from VD1, in the approximate position $(\mathrm{RA}, \mathrm{Dec})=(185.310 \mathrm{deg}, 13.675 \mathrm{deg}))$.

We used GALFIT (Peng et al. 2010) to estimate magnitudes, radii, and central surface brightness of the LSB dwarfs lying in Field D. We limit this analysis to VD1, VD2, and VD3 because they are the most prominent dwarfs of the sample and provide quantitative benchmarks to compare with SECCO 1 from exactly 


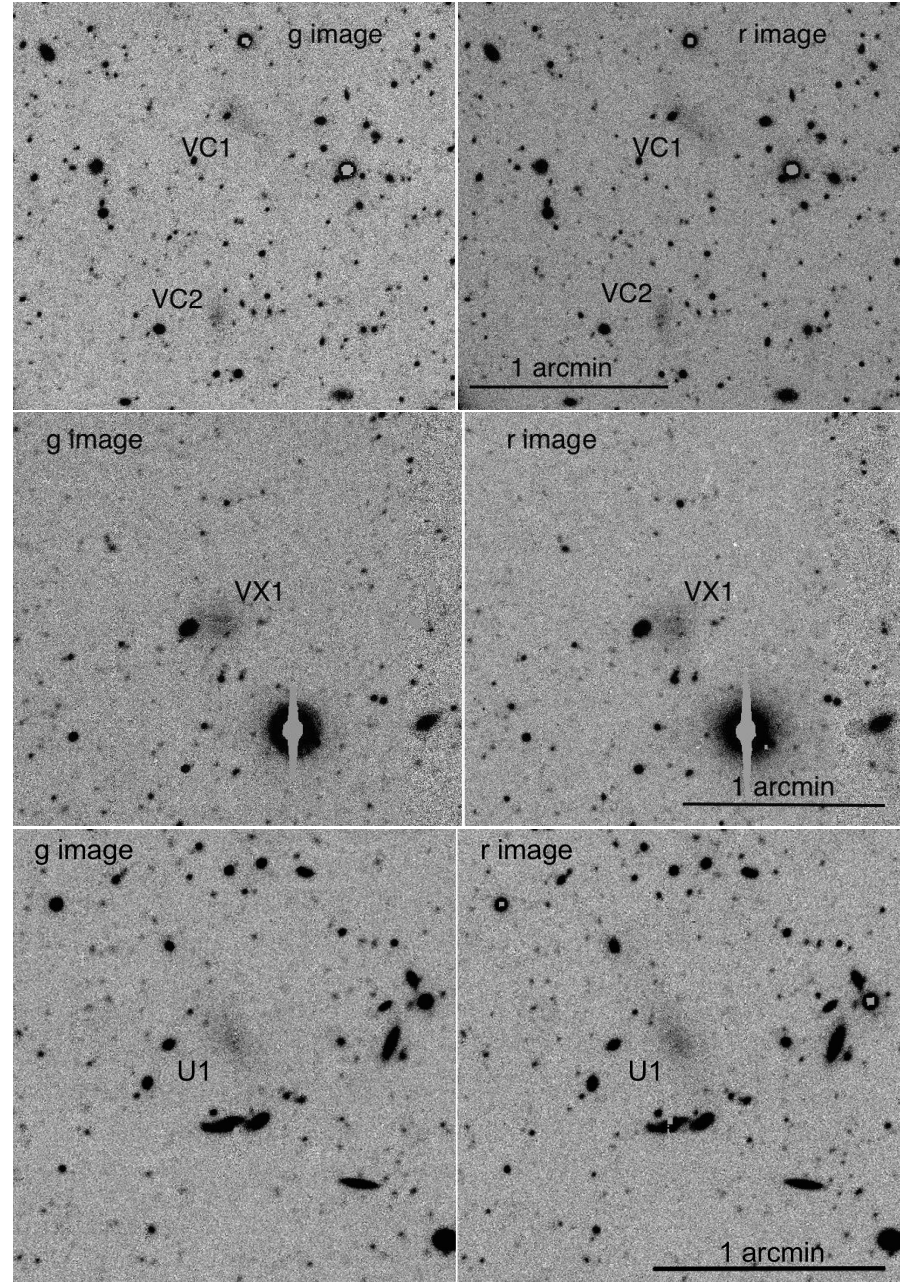

Fig. 13. Stamp images in the $g$-band (left panels) and $r$-band (right panels) of the LSB galaxies found in Field C (upper pair of images), Field X (middle pair of panels), and Field U (lower pair of panels). North is up, east to the left.

the same images. We fitted Sersic models to the $g$ and $r$ images. In a first pass we also leave the Sersic index $n_{\mathrm{s}}$ as a free parameter, then we repeated the fit keeping $n_{\mathrm{s}}$ fixed at the best-fit value of the first pass. All of the three galaxies have $n_{\mathrm{s}} \leq 1.0$ as typical of faint dwarfs in Virgo (Côté et al. 2007). Their integrated magnitudes, half-light radii, and central surface brightness, as derived with GALFIT, are listed in Table 2. We made no attempt to mask background sources, hence the reported photometry may suffer from some contamination, in particular VD1, which overlaps with a compact group of galaxies in the background (SDSSCGB22677; McConnachie et al. 2009). Moreover VD1 and VD2 lie near a corner of Field D and are partially outside of one of the two $g+r$ pairs of SECCO images of Field D, and the adjacent bright star provides a higher than average and spatially variable background. Hence the obtained surface photometry is not optimal. From the comparison of the independent measures on two images per passband for VD3, keeping a conservative attitude, we conclude that the typical uncertainty on integrated magnitudes and central SB are $\$ \pm 0.3$ mag and $\$ 20 \%$ on $R_{\mathrm{h}}$. When $D=16.5 \mathrm{Mpc}$ is assumed, VD1, VD2, and VD3 have $M_{V}=-12.4,-10.9$, and -8.2 , respectively, and they fit nicely into the $M_{V}$ versus $R_{\mathrm{h}}$ and $M_{V}$ versus $\mu_{V, 0}$ relations defined by local dwarfs (McConnachie 2012).
The $g$ and $r$ band images of the remaining LSB dwarfs listed in Table 2 are shown in Fig. 13. It is worth noting that similar to the case of Field D, the two objects identified in Field C are also very close one another. VC1 and VC2 are separated by just $1.0^{\prime}$ in the plane of the sky, corresponding to $\simeq 4.8 \mathrm{kpc}$ at the distance of Virgo. Our sample of Virgo LSB is indeed too scanty to draw any general conclusion, but it is very intriguing to note that five of the six galaxies more certainly attributable to Virgo are seen in very tight groups of two and three dwarfs.

The LSB dwarf found in Field $U$ is $\sim 4^{\circ}$ beyond the zero-velocity contour of the Virgo cluster derived by Karachentsev \& Nosonova $\left(2010, \sim 17^{\circ}\right)$, hence it cannot be considered as a member of Virgo. However it is located in the plane of the sky in the middle between two galaxy groups (Leo I and Leo II/NGC 3607) that lie at similar distance; if it is associated with such groups, as seems likely, U1 is also a dwarf galaxy similar to the others listed in Table 2.

The detection of the faint LSB dwarfs discussed in this section fully support the results presented above, providing an independent empirical validation of the search by visual inspection we performed in Paper I and of the quantitative sensitivity analysis presented here.

\subsection{Comparison with SECCO 1}

Figure 14 allows a direct comparison of the size, brightness, morphology, and colours of SECCO 1 and of the LSB dwarfs identified in the same SECCO images (Field D). The (reasonable) assumption that all the considered stellar systems lie within the Virgo cluster makes the comparison especially insightful. On the left panel of the image we highlighted the main body of SECCO 1 (Bellazzini et al. 2015b) as well as the possible secondary body identified by Sand et al. (2015). We highlighted with an ellipse a possible additional grouping of blue stars that may be associated with the system; a similar swarm of faint blue stars is also seen just to the east of the secondary body. If these structures are indeed physically associated, then SECCO 1 is a system even more complex and anomalous than already believed (Adams et al. 2015), and a tidal (Duc 2012) or ram-pressure (Fumagalli et al. 2011; Yoshida et al. 2012) stripping phenomenon would gain support as a hypothesis for its origin (see Bellazzini et al. 2015b).

The comparison with the Virgo dSphs lying in the same image, in particular with the faintest and most diffuse dSph, VD2, leads to some interesting conclusions:

- Any diffuse component associated with SECCO 1 should be significantly fainter than the central SB of VD2, i.e. $\mu_{V} \ll 26.5 \mathrm{mag} \operatorname{arcsec}^{2}$. The comparison with our simulated images consistently indicates that it should be in fact $\mu_{V} \ll 27.0 \mathrm{mag} \operatorname{arcsec}^{2}$.

- Any diffuse component associated with SECCO 1 is significantly bluer than the dSphs. Indeed some very feeble fuzzy blue light is seen within the circle enclosing the main body, especially to the east of the brightest sources and in the middle of the secondary body.

\section{Summary and conclusions}

We complemented the search for stellar counterparts in 25 selected ALFALFA UCHVCs (A13), performed in Paper I, with a wide set of experiments with synthetic dwarf galaxies to quantitatively assess the sensitivity of the SECCO survey. We explored a grid of models of old and metal-poor stellar systems in the 


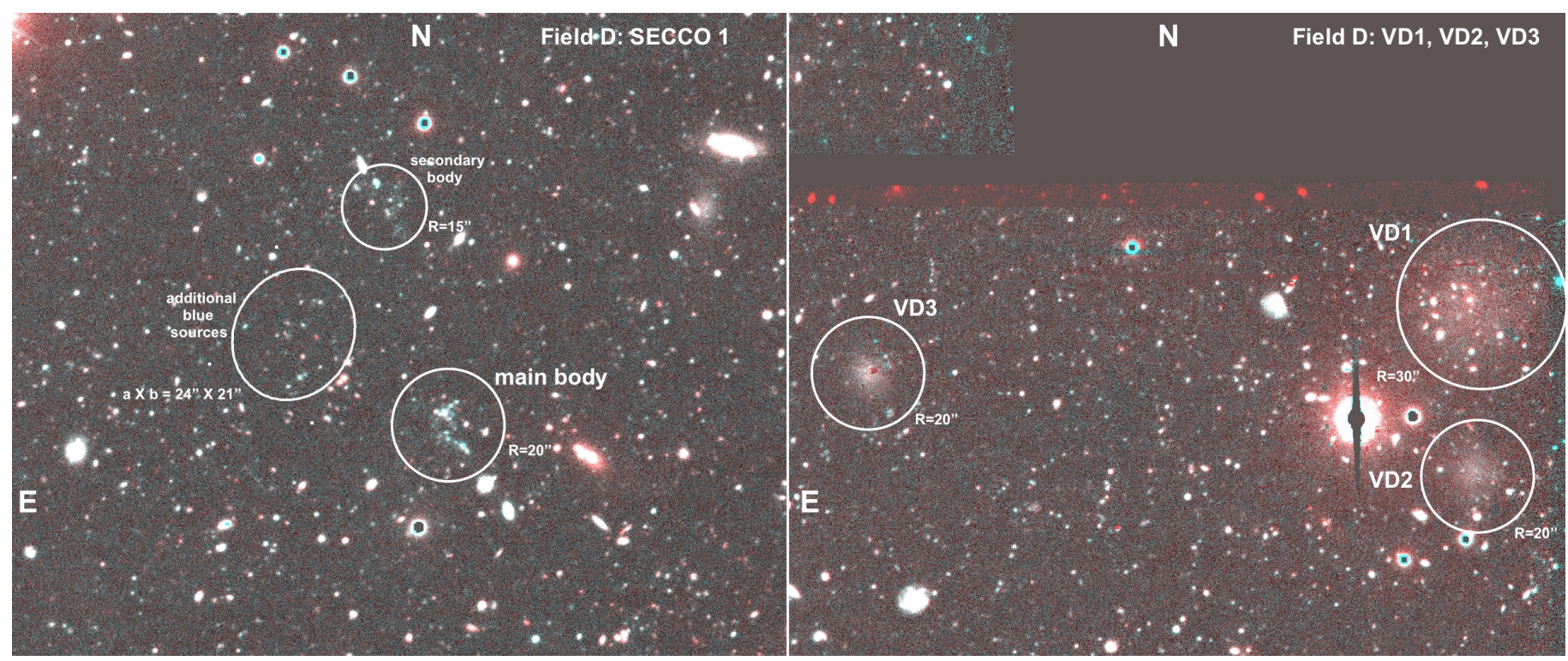

Fig. 14. RGB colour stacked images of SECCO Field D centred on SECCO 1 (left panel) and VD1, VD2, and VD3 (right panel). The images were obtained using the $r$-band image for the $\mathrm{R}$ channel and the $g$-band image for the $\mathrm{G}$ and $\mathrm{B}$ channels. The most remarkable structures were labelled and enclosed with circles/ellipses whose size is reported.

range $-5.0 \leq M_{V} \leq-10.0$. The stars of the synthetic dwarfs are distributed according to an exponential profile with three different $R_{\mathrm{h}}$ values spanning the $\pm 1 \sigma_{\log R_{\mathrm{h}}}$ range of the mean $M_{V}$ vs. $R_{\mathrm{h}}$ relation as modelled by Brasseur et al. (2011, based on local galaxies). We analysed the sensitivity of stellar density maps in the range $0.25 \mathrm{Mpc} \leq D \leq 2.5 \mathrm{Mpc}$ and the sensitivity of visual inspection of the images in the range $0.25 \mathrm{Mpc} \leq D \leq 5.0 \mathrm{Mpc}$.

We fully confirm and extend the results of Paper I. In particular, we can now safely conclude the following:

1. There is no dwarf galaxy with $R_{\mathrm{h}} \leq \bar{R}_{\mathrm{h}}\left(M_{v}\right)+1 \sigma_{\log R_{\mathrm{h}}}$ and $M_{V} \leq-10.0$, within $D \leq 5.0 \mathrm{Mpc}$, associated with any of the 25 A13 UCHVCs studied by SECCO.

2. There is no dwarf galaxy with $R_{\mathrm{h}} \leq \bar{R}_{\mathrm{h}}\left(M_{v}\right)+1 \sigma_{\log R_{\mathrm{h}}}$ and $M_{V} \leq-9.0$ within $D \leq 1.0 \mathrm{Mpc}$ associated with any of the 25 A13 UCHVCs studied by SECCO.

3. If we consider only dwarfs with $R_{\mathrm{h}} \leq \bar{R}_{\mathrm{h}}\left(M_{v}\right)$, there is no galaxy with $M_{V} \leq-9.0$ within $D \leq 2.0 \mathrm{Mpc}$ (as well as no galaxy with $M_{V} \leq-8.0$ within $D \leq 1.5 \mathrm{Mpc}$ ) associated with any of the 25 A13 UCHVCs studied by SECCO.

4. If we consider the nine SECCO fields with the best data quality (namely, A, G, B, Y, R, K, U, C, L; see Paper I) we can conclude that there is no dwarf with $M_{V} \leq-8.0$ within $D \leq 2.5 \mathrm{Mpc}$ (as well as no galaxy with $M_{V} \leq-7.0$ within $D \leq 1.5 \mathrm{Mpc}$ ) associated with the corresponding A13 UCHVCs.

Thus we fully confirm the lack of $M_{V} \leq-8.0$ local counterparts to ALFALFA UCHVCs already mentioned in Paper I and, later, confirmed on the whole A13 sample but with shallower and less homogeneous data by Sand et al. (2015).

In Fig. 15 we provide a complementary view of the summary of our results presented in Figs. 8 and 9, which may help express our sensitivity limits in a more synthetic form, in the distance versus surface brightness plane. In "Best case" observations, we can detect as $\geq 5 \sigma$ over-densities dwarfs with $\mu_{V, \mathrm{~h}} \leq 30.0 \mathrm{mag} / \operatorname{arcsec}^{2}$ out to $D \leq 1.5 \mathrm{Mpc}$, and those with $\mu_{V, \mathrm{~h}} \leq 29.5 \mathrm{mag} / \operatorname{arcsec}^{2}$ out to $D \leq 2.5 \mathrm{Mpc}$. In "Worst case" observations, we can detect as $\geq 5 \sigma$ over-densities dwarfs with

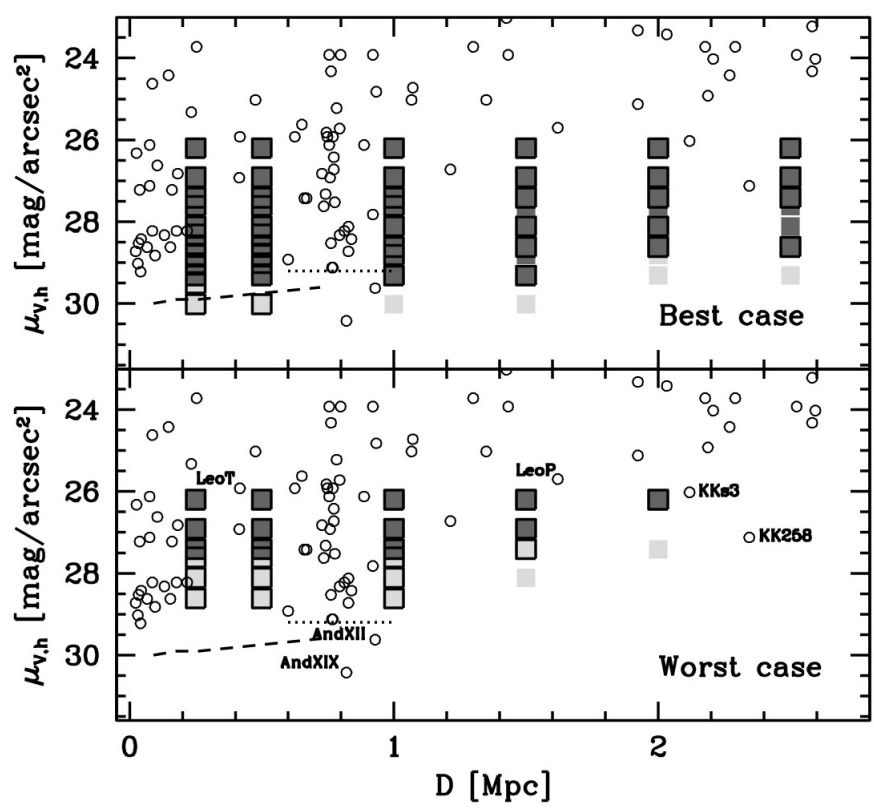

Fig. 15. Sensitivity of density maps for "Best" (upper panel) and "Worst" (lower panel) cases in the distance vs. surface brightness plane. The symbols are the same as in Fig. 8. In particular, light open circles are known dwarf galaxies in the LV, from McConnachie (2012). Central surface brightness values reported in the McConnachie (2012) catalogue were transformed into $\mu_{V, \mathrm{~h}}$ assuming exponential profiles, an acceptable approximation in the considered range of luminosity (Mateo 1998). We labelled six remarkable systems. The dashed line indicates the SDSS detection limit as a function of distance for resolved stellar systems from Koposov et al. (2008, their Table 3); the dotted line is the detection limit of the PAndAS survey (Ibata et al. 2007) in the surroundings of M31, from Brasseur et al. (2011).

$\mu_{V, \mathrm{~h}} \leq 28.8 \mathrm{mag} / \operatorname{arcsec}^{2}$ out to $D \leq 1.0 \mathrm{Mpc}$, and those with $\mu_{V, \mathrm{~h}} \leq 27.6 \mathrm{mag} / \operatorname{arcsec}^{2}$ out to $D \leq 2.5 \mathrm{Mpc}$. More specifically, the comparison with the distribution of known dwarfs in the LV 
(from McConnachie 2012) shows that, for all the surveyed fields we would have detected any galaxy lying in range of distances and surface brightness inhabited by known LV dwarfs (except perhaps And XII, And XIX, KKs3, and KK258, with Worst case observations), independent of the quality of the available data. Moreover, our sensitivity region extends into a range of low surface brightness and large distances (for $1.0 \mathrm{Mpc} \lesssim D \lesssim 2.5 \mathrm{Mpc}$ ) that appears as largely unexplored at present.

In particular, Fig. 15 shows that our survey is sensitive to dwarfs much fainter than the prototypical gas-rich faint dwarfs Leo $\mathrm{T}$ and Leo $\mathrm{P}$.

It is important to stress here that $M_{V}=-10.0$ corresponds to $8.5 \times 10^{5} L_{V, \odot}, M_{V}=-9.0$ to $3.4 \times 10^{5} L_{V, \odot}, M_{V}=-8.0$ to $1.3 \times 10^{5} L_{V, \odot}$, and $M_{V}=-7.0$ to $5 \times 10^{4} L_{V, \odot}$. Since starforming galaxies have stellar mass-to-light ratios $M / L \leq 1.0$, these translate into very strong constraints in terms of total stellar mass of any stellar counterpart that went undetected in SECCO. For example, assuming a distance of $1.0 \mathrm{Mpc}$ A13 concluded that ALFALFA UCHVCs span a range of $\mathrm{HI}$ masses $10^{5} M_{\odot} \lesssim M_{\mathrm{HI}} \lesssim 10^{6} M_{\odot}$. This would imply that any associated stellar system not detected in SECCO would typically have $M_{\mathrm{HI}} / M_{*} \gtrsim 10$, a value that is significantly higher than that found in normal star-forming dwarfs (see e.g. Cannon et al. 2015; Bellazzini et al. 2015b; Sand et al. 2015) but typical of extremely metal-poor galaxies (XMPs; Filho et al. 2013).

In conclusion, the results of our very deep and homogeneous survey concerning local counterparts of high velocity clouds are fully in line with various unsuccessful attempts performed in the last two decades (see e.g. Simon \& Blitz 2002; Willman et al. 2002; Hopp et al. 2003, 2007; Siegel et al. 2005, and references therein). The novelty is that, on the other hand, some more distant counterpart begins to emerge (Paper I, Bellazzini et al. 2015b), especially among the GALFA-HI candidates (Sand et al. 2015). The follow up of these counterparts and further searches in better defined samples of candidates may provide crucial insight into the census and the evolution of very low-mass, starforming dwarfs.

Acknowledgements. We are grateful to the anonymous referee for providing insightful comments and precious suggestions for a clearer and more meaningful presentation of the results of this analysis. We acknowledge the support from the LBT-Italian Coordination Facility for the execution of observations, data distribution, and reduction. M.B. acknowledges the financial support from PRIN MIUR 2010-2011 project "The Chemical and Dynamical Evolution of the Milky Way and Local Group Galaxies", prot. 2010LY5N2T. G. Battaglia gratefully acknowledges support through a Marie-Curie action Intra European Fellowship, funded from the European Union Seventh Framework Program (FP7/2007-2013) under Grant agreement number PIEF-GA-2010-274151, as well as the financial support by the Spanish Ministry of Economy and Competitiveness (MINECO) under the Ramón y Cajal Program (RYC-2012-11537). This research has made use of the SIMBAD database, operated at CDS, Strasbourg, France. This research has made use of the NASA/IPAC Extragalactic Database (NED), which is operated by the Jet Propulsion Laboratory, California Institute of Technology, under contract with the National Aeronautics and Space Administration. This research has made use of NASA's Astrophysics Data System. This research make use of SDSS data. Funding for the SDSS and SDSS- II has been provided by the Alfred P. Sloan Foundation, the Participating Institutions, the National Science Foundation, the US Department of Energy, the National Aeronautics and Space Administration, the Japanese Monbukagakusho, the Max Planck Society, and the Higher Education Funding Council for England. The SDSS Web Site is http:www.sdss.org. The SDSS is managed by the Astrophysical Research Consortium for the Participating Institutions. The Participating Institutions are the American Museum of Natural History, Astrophysical Institute Potsdam, University of Basel, University of Cambridge, Case Western Reserve University, University of Chicago, Drexel University, Fermilab, the Institute for Advanced Study, the Japan Participation Group, Johns Hopkins University, the Joint Institute for Nuclear Astrophysics, the Kavli Institute for
Particle Astrophysics and Cosmology, the Korean Scientist Group, the Chinese Academy of Sciences (LAMOST), Los Alamos National Laboratory, the MaxPlanck-Institute for Astronomy (MPIA), the Max-Planck- Institute for Astrophysics (MPA), New Mexico State University, Ohio State University, University of Pittsburgh, University of Portsmouth, Princeton University, the United States Naval Observatory, and the University of Washington.

\section{References}

Adams, E. K., Giovanelli, R., \& Haynes, M. P. 2013, ApJ, 768, 77 (A13) Adams, E. K., Cannon, J. M., Rhode, K. L., et al. 2015, A\&A, 573, L3 Bellazzini, M., Beccari, G., Battaglia, G., et al. 2015a, A\&A, 575, A126 (Paper I) Bellazzini, M., Magrini, L., Mucciarelli, A., et al. 2015b, ApJ, 800, L15 Belokurov, V. 2013, New Astron. Rev., 57, 100

Brasseur, C. M., Martin, N. F., Maccio', A. V., Rix, H.-W., \& Kanf, X. 2011, ApJ, 743, 179

Bressan, A., Marigo, P., Girardi, L., et al. 2012, MNRAS, 427, 127

Caldwell, N. 2006, ApJ, 651, 822

Cannon, J. M., Martinkus, C. P., \& Leisman, L. 2015, AJ, 149, 72

Ciotti, L., \& Bertin, G. 1999, A\&A, 352, 447

Côté, P., Ferrarese, L., Jordán, A., et al. 2008, IAU Symp. 245, eds. M. Bureau, E. Athanassoula, \& B. Barbuy, 395

Davies, J. I., Davies, L. J. M., \& Keenan, O. C. 2016, MNRAS, 456, 1607

Donovan Meyer, J., Peek, J. E. G., Putman, M., \& Grchevich, J. 2016, ApJ, 808, 136

Duc, P. A. 2012, in Dwarf Galaxies: Keys to Galaxy Formation and Evolution, eds. P. Papaderos, S. recchi, \& G. Hensler, Astrophys. Space Sci. Proc. (Berlin Heidelberg: Springer-Verlag), 305

Ferrarese, L., Côté, P., \& Cuillandre, J.-C. 2012, ApJS, 200, 4

Filho, M. E., Winkel, B., Sánchez-Almeida, J., et al. 2013, A\&A, 558, A18

Fumagalli, M., Gavazzi, G., Scaramella, R., \& Franzetti, P. 2011, A\&A, 528, A46

Giallongo, E., Ragazzoni, R., \& Grazian, A. 2008, A\&A, 482, 349

Giovanelli, R., Haynes, M. P., Kent, B. R., et al. 2007, AJ, 133, 2583

Giovanelli, R., Haynes, M. P., \& Adams, E. 2013, AJ, 146, 15

Girardi, L., Groenewegen, M. A. T., Hatziminaoglu, E., \& da Costa, L. 2005, A\&A, 436, 895

Ibata, R., Martin, N. F., Irwin, M., et al. 2007, ApJ, 671, 1591

Irwin, M. J., Belokurov, V., Evans, N. W., et al. 2007, ApJ, 656, L13

Hopp, U., Schulte-Ladbeck, R. E., \& Kerp, J. 2003, MNRAS, 339, 33

Hopp, U., Schulte-Ladbeck, R. E., \& Kerp, J. 2007, MNRAS, 374, 1164

Karachentsev, I. D., \& Nasonova, O. G. 2010, MNRAS, 405, 1075

Kauffman, G., White, S. D. M., \& Guiderdoni, B. 1993, MNRAS, 264, 201

Kim, S., Rey, S.-C., Jerjen, H., et al. 2014, ApJS, 215, 22

Klypin, A., Kravtsov, A. V., Valenzuela, O., \& Prada, F. 1999, ApJ, 522, 82

Koposov, S. E., Belokurov, V., Evans, N. W., et al. 2008, ApJ, 686, 279

Kravtsov, A. V., Gnedin, O. Y., \& Klypin, A. A. 2004, ApJ, 609, 482

Martin, N. F., de Jong, J. T.A, \& Rix, H.-W. 2008, ApJ, 684, 1075

Mateo, M. 1998, ARA\&A, 36, 435

Mei, S., Blakeslee, J. P., Côté, P., et al. 2007, ApJ, 655, 144

Mihos, C., Durrell, P. R., Ferrarese, L., et al. 2015, ApJ, 809, L21

McConnachie, A. 2012, AJ, 144, 4

McConnachie, A., Patton, D. R., Ellison, S. L., \& Simard, L. 2009, MNRAS, 395,255

McQuinn, K. D. W., Skillman, E. D., Berg, D. A., et al. 2013, AJ, 146, 145

Moore, B., Ghigna, S., Governato, F., et al. 1999, ApJ, 524, L19

Peng, C. Y., Ho, L. C., Impey, C. D., \& Rix., H.-W. 2010, AJ, 139, 2097

Phillips, K. J. H., Parker, Q. A., Scwartzenberg, J. M., \& Jones, J. B. 1998, ApJ, 439, L59

Ricotti, M. 2009, MNRAS, 392, L45

Sabatini, S., Davies, J., Scaramella, R., et al. 2003, MNRAS, 341, 981

Sand, D. J., Crnojevic, D., Bennet, P., et al. 2015, ApJ, 806, 95

Saul, D. R., Peek, J. E. G., Grchevich, J., et al. 2012, ApJ, 758, 44

Sawala, T., Frenk, C. S., Crain, R. A., et al. 2013, MNRAS, 431, 1366

Siegel, M. H., Majewski, S. R., Gallart, C., et al. 2005, ApJ, 623, 181

Simon, J. D., \& Blitz, L. 2002, ApJ, 574, 726

Stetson, P. B. 1987, PASP, 99, 191

Tollerud, E. J., Geha, M. C., Grcevich, J., Putman, M. E., \& Stern, D. 2015, ApJ, 798, L21

Tolstoy, E., Hill, V., \& Tosi, M. 2009, ARA\&A, 47, 371

Yoshida, M., Yagi, M., Komiyama, Y., et al. 2012, ApJ, 749, 43

Weisz, D. R., Dolphin, A. E., Skillman, E. D., et al. 2014, ApJ, 789, 147

Willman, B., Dalcanton, J., Ivezić, Z., et al. 2002, AJ, 123, 848 
G. Beccari et al.: The StEllar Counterparts of COmpact high velocity clouds (SECCO) survey. II.

\section{Appendix A: Sensitivity of density maps: a summary table}

In Table A.1 we summarise the results of our experiments for the detection synthetic dwarf galaxies with density maps described in Sect. 3 and illustrated in Figs. 8 and 9.

Table A.1. Classification of the over-density detections.

\begin{tabular}{|c|c|c|c|c|c|c|c|c|}
\hline$M_{V}$ & $k^{a}$ & $D$ & $B_{10}$ & $B_{5}$ & $B_{\mathrm{CMD}}$ & $I_{10}$ & $I_{5}$ & $I_{\mathrm{CMD}}$ \\
\hline-9.0 & -1 & 0.25 & 1 & 1 & 1 & 1 & 1 & 1 \\
\hline-9.0 & -1 & 0.50 & 1 & 1 & 1 & 1 & 1 & 1 \\
\hline-9.0 & -1 & 1.00 & 1 & 1 & 1 & 1 & 1 & 1 \\
\hline-9.0 & -1 & 1.50 & 1 & 1 & 1 & 1 & 1 & 1 \\
\hline-9.0 & -1 & 2.00 & 1 & 1 & 1 & 1 & 1 & 1 \\
\hline-9.0 & -1 & 2.50 & 1 & 1 & 1 & 0 & 0 & 0 \\
\hline-9.0 & 0 & 0.25 & 1 & 1 & 1 & 1 & 1 & 1 \\
\hline-9.0 & 0 & 0.50 & 1 & 1 & 1 & 1 & 1 & 1 \\
\hline-9.0 & 0 & 1.00 & 1 & 1 & 1 & 1 & 1 & 1 \\
\hline-9.0 & 0 & 1.50 & 1 & 1 & 1 & 0 & 1 & 1 \\
\hline-9.0 & 0 & 2.00 & 1 & 1 & 1 & 0 & 1 & 0 \\
\hline-9.0 & 0 & 2.50 & 1 & 1 & 1 & 0 & 0 & 0 \\
\hline-9.0 & +1 & 0.25 & 1 & 1 & 1 & 0 & 1 & 1 \\
\hline-9.0 & +1 & 0.50 & 1 & 1 & 1 & 0 & 1 & 1 \\
\hline-9.0 & +1 & 1.00 & 1 & 1 & 1 & 0 & 1 & 1 \\
\hline-9.0 & +1 & 1.50 & 1 & 1 & 1 & 0 & 0 & 0 \\
\hline-9.0 & +1 & 2.00 & 1 & 1 & 1 & 0 & 0 & 0 \\
\hline-9.0 & +1 & 2.50 & 1 & 1 & 1 & 0 & 0 & 0 \\
\hline-8.0 & -1 & 0.25 & 1 & 1 & 1 & 1 & 1 & 1 \\
\hline-8.0 & -1 & 0.50 & 1 & 1 & 1 & 1 & 1 & 1 \\
\hline-8.0 & -1 & 1.00 & 1 & 1 & 1 & 1 & 1 & 1 \\
\hline-8.0 & -1 & 1.50 & 1 & 1 & 1 & 1 & 1 & 1 \\
\hline-8.0 & -1 & 2.00 & 1 & 1 & 1 & 0 & 0 & 0 \\
\hline-8.0 & -1 & 2.50 & 1 & 1 & 1 & 0 & 0 & 0 \\
\hline-8.0 & 0 & 0.25 & 1 & 1 & 1 & 0 & 1 & 1 \\
\hline-8.0 & 0 & 0.50 & 1 & 1 & 1 & 0 & 1 & 1 \\
\hline-8.0 & 0 & 1.00 & 1 & 1 & 1 & 0 & 1 & 1 \\
\hline-8.0 & 0 & 1.50 & 1 & 1 & 1 & 0 & 1 & 0 \\
\hline-8.0 & 0 & 2.00 & 1 & 1 & 1 & 0 & 0 & 0 \\
\hline-8.0 & 0 & 2.50 & 1 & 1 & 0 & 0 & 0 & 0 \\
\hline-8.0 & +1 & 0.25 & 1 & 1 & 1 & 0 & 0 & 0 \\
\hline-8.0 & +1 & 0.50 & 1 & 1 & 1 & 0 & 0 & 0 \\
\hline-8.0 & +1 & 1.00 & 1 & 1 & 1 & 0 & 0 & 0 \\
\hline-8.0 & +1 & 1.50 & 1 & 1 & 1 & 0 & 0 & 0 \\
\hline-8.0 & +1 & 2.00 & 0 & 1 & 0 & 0 & 0 & 0 \\
\hline-8.0 & +1 & 2.50 & 0 & 1 & 0 & 0 & 0 & 0 \\
\hline-7.0 & -1 & 0.25 & 1 & 1 & 1 & 0 & 1 & 1 \\
\hline-7.0 & -1 & 0.50 & 1 & 1 & 1 & 0 & 1 & 1 \\
\hline-7.0 & -1 & 1.00 & 1 & 1 & 1 & 0 & 1 & 1 \\
\hline-7.0 & -1 & 1.50 & 1 & 1 & 0 & 0 & 0 & 0 \\
\hline-7.0 & -1 & 2.00 & 1 & 1 & 0 & 0 & 0 & 0 \\
\hline-7.0 & -1 & 2.50 & 1 & 1 & 0 & 0 & 0 & 0 \\
\hline-7.0 & 0 & 0.25 & 1 & 1 & 1 & 0 & 0 & 0 \\
\hline-7.0 & 0 & 0.50 & 1 & 1 & 1 & 0 & 0 & 0 \\
\hline-7.0 & 0 & 1.00 & 1 & 1 & 1 & 0 & 0 & 0 \\
\hline
\end{tabular}

Notes. ${ }^{(a)}$ Indicates compact $(k=-1)$, average $(k=0)$ or diffuse $(k=+1)$ models, where $R_{\mathrm{h}}=\bar{R}_{\mathrm{h}}\left(M_{v}\right)+k \sigma_{\log R_{\mathrm{h}}} . B_{10}\left(I_{10}\right)=1$ indicates a $\geq 10 \sigma$ detection in Best (Worst) case observations (Field B and Field I, respectively). $B_{5}\left(I_{5}\right)=1$ indicates a $\geq 5 \sigma$ and $<10 \sigma$ detection in Best (Worst) case observations (Field B and Field I, respectively). $B_{\mathrm{CMD}}\left(I_{\mathrm{CMD}}\right)=1$ indicates that the inspection of the $\mathrm{CMD}$ provide significant support to the classification of the detected over-density as a dwarf galaxy.
Table A.1. continued.

\begin{tabular}{ccccccccc}
\hline \hline$M_{V}$ & $k^{a}$ & $D$ & $B_{10}$ & $B_{5}$ & $B_{\mathrm{CMD}}$ & $I_{10}$ & $I_{5}$ & $I_{\mathrm{CMD}}$ \\
\hline-7.0 & 0 & 1.50 & 1 & 1 & 0 & 0 & 0 & 0 \\
-7.0 & 0 & 2.00 & 0 & 1 & 0 & 0 & 0 & 0 \\
-7.0 & 0 & 2.50 & 0 & 0 & 0 & 0 & 0 & 0 \\
-7.0 & +1 & 0.25 & 0 & 1 & 1 & 0 & 0 & 0 \\
-7.0 & +1 & 0.50 & 0 & 1 & 1 & 0 & 0 & 0 \\
-7.0 & +1 & 1.00 & 0 & 1 & 0 & 0 & 0 & 0 \\
-7.0 & +1 & 1.50 & 0 & 1 & 0 & 0 & 0 & 0 \\
-7.0 & +1 & 2.00 & 0 & 0 & 0 & 0 & 0 & 0 \\
-7.0 & +1 & 2.50 & 0 & 0 & 0 & 0 & 0 & 0 \\
-6.0 & -1 & 0.25 & 1 & 1 & 1 & 0 & 0 & 0 \\
-6.0 & -1 & 0.50 & 0 & 1 & 1 & 0 & 0 & 0 \\
-6.0 & -1 & 1.00 & 0 & 1 & 0 & 0 & 0 & 0 \\
-6.0 & -1 & 1.50 & 0 & 1 & 0 & 0 & 0 & 0 \\
-6.0 & -1 & 2.00 & 0 & 0 & 0 & 0 & 0 & 0 \\
-6.0 & -1 & 2.50 & 0 & 0 & 0 & 0 & 0 & 0 \\
-6.0 & 0 & 0.25 & 0 & 1 & 1 & 0 & 0 & 0 \\
-6.0 & 0 & 0.50 & 0 & 0 & 0 & 0 & 0 & 0 \\
-6.0 & 0 & 1.00 & 0 & 0 & 0 & 0 & 0 & 0 \\
-6.0 & 0 & 1.50 & 0 & 0 & 0 & 0 & 0 & 0 \\
-6.0 & 0 & 2.00 & 0 & 0 & 0 & 0 & 0 & 0 \\
-6.0 & 0 & 2.50 & 0 & 0 & 0 & 0 & 0 & 0 \\
-6.0 & +1 & 0.25 & 0 & 0 & 0 & 0 & 0 & 0 \\
-6.0 & +1 & 0.50 & 0 & 0 & 0 & 0 & 0 & 0 \\
-6.0 & +1 & 1.00 & 0 & 0 & 0 & 0 & 0 & 0 \\
-6.0 & +1 & 1.50 & 0 & 0 & 0 & 0 & 0 & 0 \\
-6.0 & +1 & 2.00 & 0 & 0 & 0 & 0 & 0 & 0 \\
-6.0 & +1 & 2.50 & 0 & 0 & 0 & 0 & 0 & 0 \\
-5.0 & -1 & 0.25 & 0 & 1 & 1 & 0 & 0 & 0 \\
-5.0 & -1 & 0.50 & 0 & 1 & 1 & 0 & 0 & 0 \\
-5.0 & -1 & 1.00 & 0 & 1 & 0 & 0 & 0 & 0 \\
-5.0 & -1 & 1.50 & 0 & 0 & 0 & 0 & 0 & 0 \\
-5.0 & -1 & 2.00 & 0 & 0 & 0 & 0 & 0 & 0 \\
-5.0 & -1 & 2.50 & 0 & 0 & 0 & 0 & 0 & 0 \\
\hline & & & & & & & &
\end{tabular}

\section{Appendix B: A small atlas of synthetic dwarfs}

In this appendix we provide images of synthetic galaxies exploring the most relevant cases for establishing the sensitivity limits of our survey. We feel that this small atlas of synthetic dwarfs can be of general utility to interpret images of candidate dwarfs obtained with $8-\mathrm{m}$ class telescopes. We simulate the galaxy image for five different distances $(D=0.5,1.0,2.0,3.0$, and $5.0 \mathrm{Mpc})$ for a given galaxy model (identified by its $M_{V}$ and $R_{\mathrm{h}}$ values) and for a given observational condition (Field B: best conditions, Field I: worst conditions). This is represented, in the following, with six-panel figures with the stamp images of the given model for the five considered distances and a sixth panel showing the original empty image. In each of the six panels, the characteristics of the model as well as the considered value of the distance are clearly reported. A circle with radius equal to $R_{\mathrm{h}}$ is superimposed and its value in arcsec is reported. Usually the images in the last four stamp-size panels have the same scale, while the first two are zoomed out to include the radius $=R_{\mathrm{h}}$ circle. The lower set of panels in Fig. B.1 shows that a $M_{V}=-10.0$ would have been visually detected in SECCO images even in fields with the worst image quality and for diffuse models, i.e. dwarfs with $R_{\mathrm{h}}=\bar{R}_{\mathrm{h}}\left(M_{v}\right)+1 \sigma_{\log R_{\mathrm{h}}}$. 


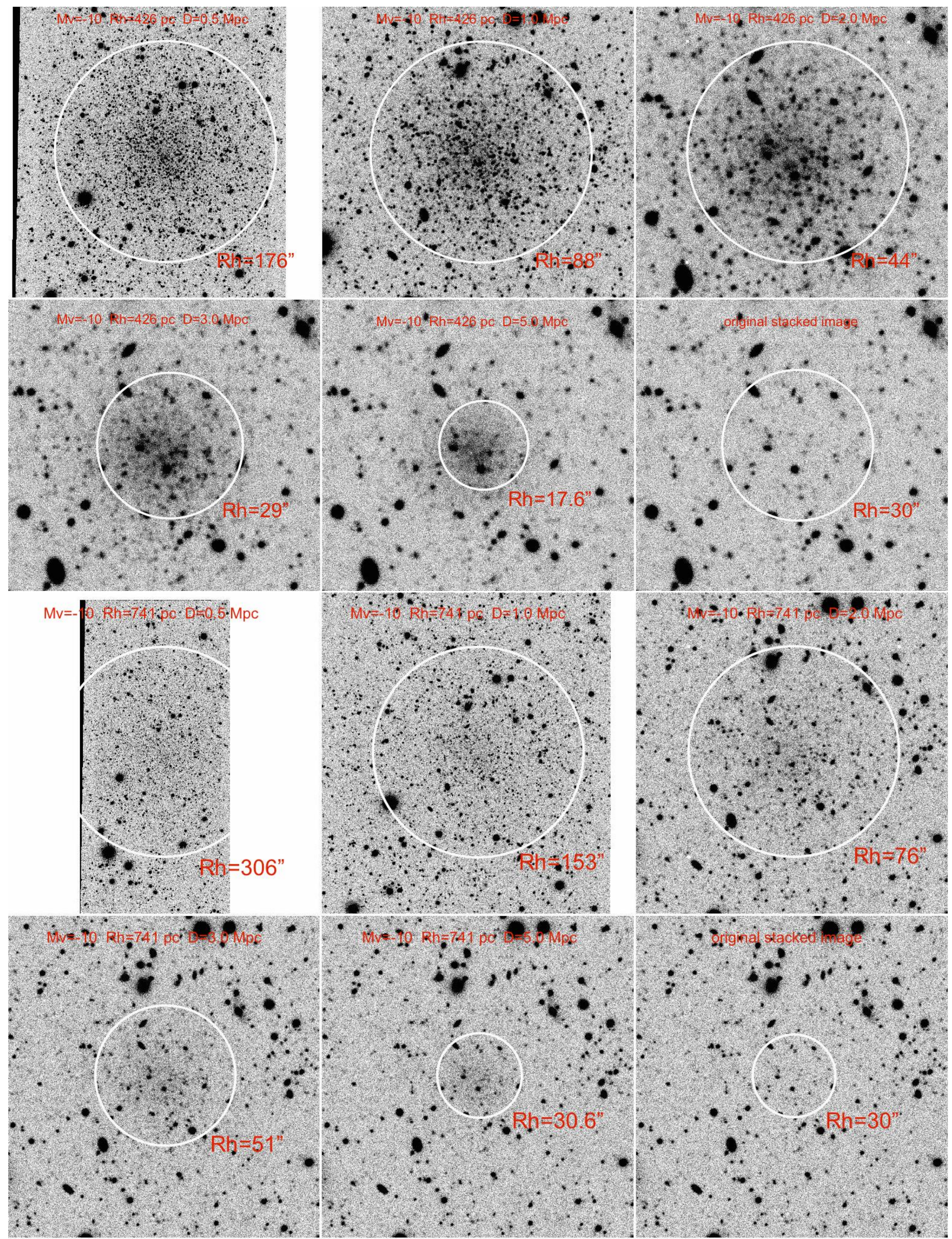

Fig. B.1. Worst case observations, $M_{V}=-10.0$, average (upper series of panels) and diffuse models (lower series of panels). 
G. Beccari et al.: The StEllar Counterparts of COmpact high velocity clouds (SECCO) survey. II.

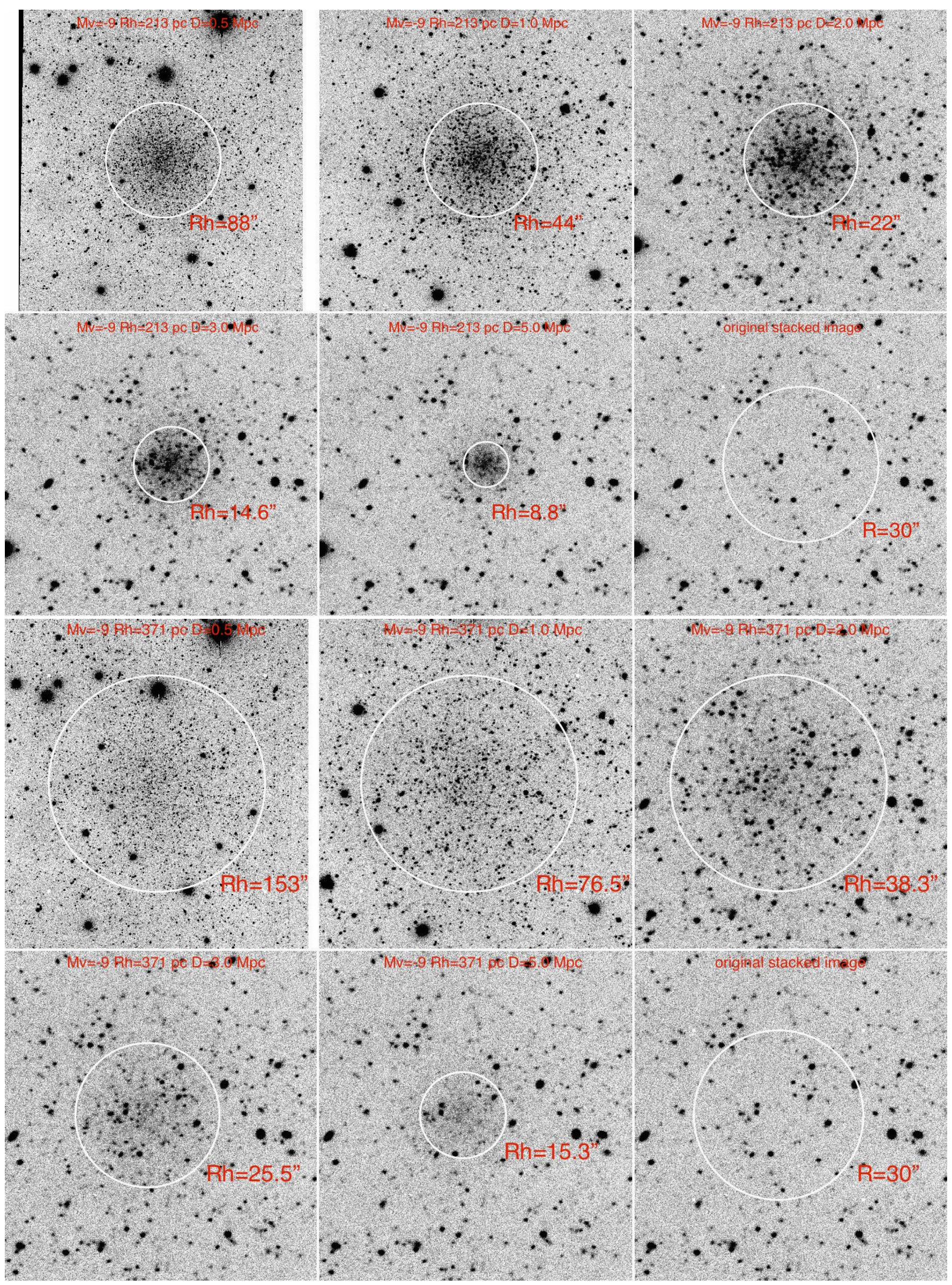

Fig. B.2. Best case observations, $M_{V}=-9.0$, compact (upper series of panels) and average models (lower series of panels). 


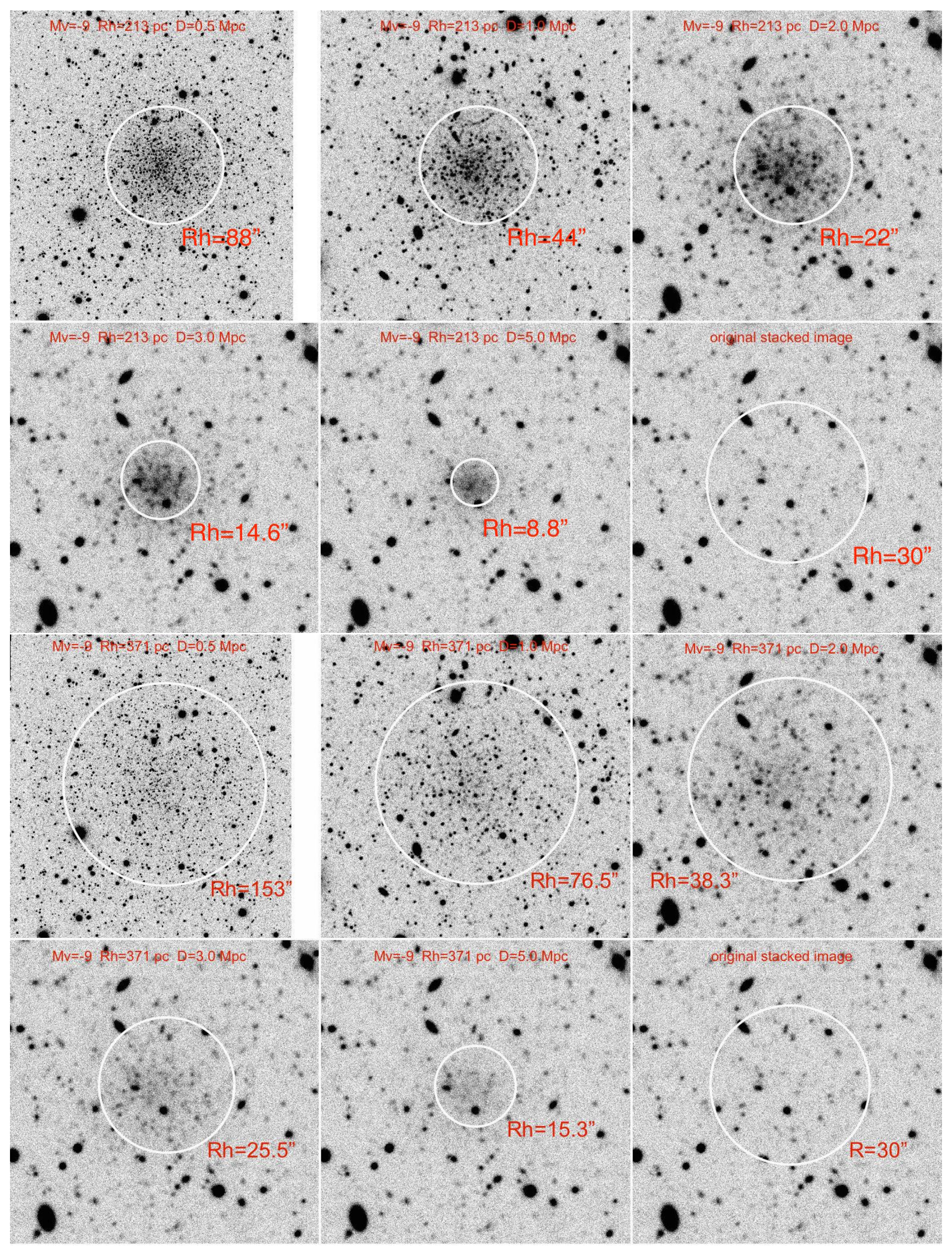

Fig. B.3. Worst case observations, $M_{V}=-9.0$, compact (upper series of panels) and average models (lower series of panels). 
G. Beccari et al.: The StEllar Counterparts of COmpact high velocity clouds (SECCO) survey. II.

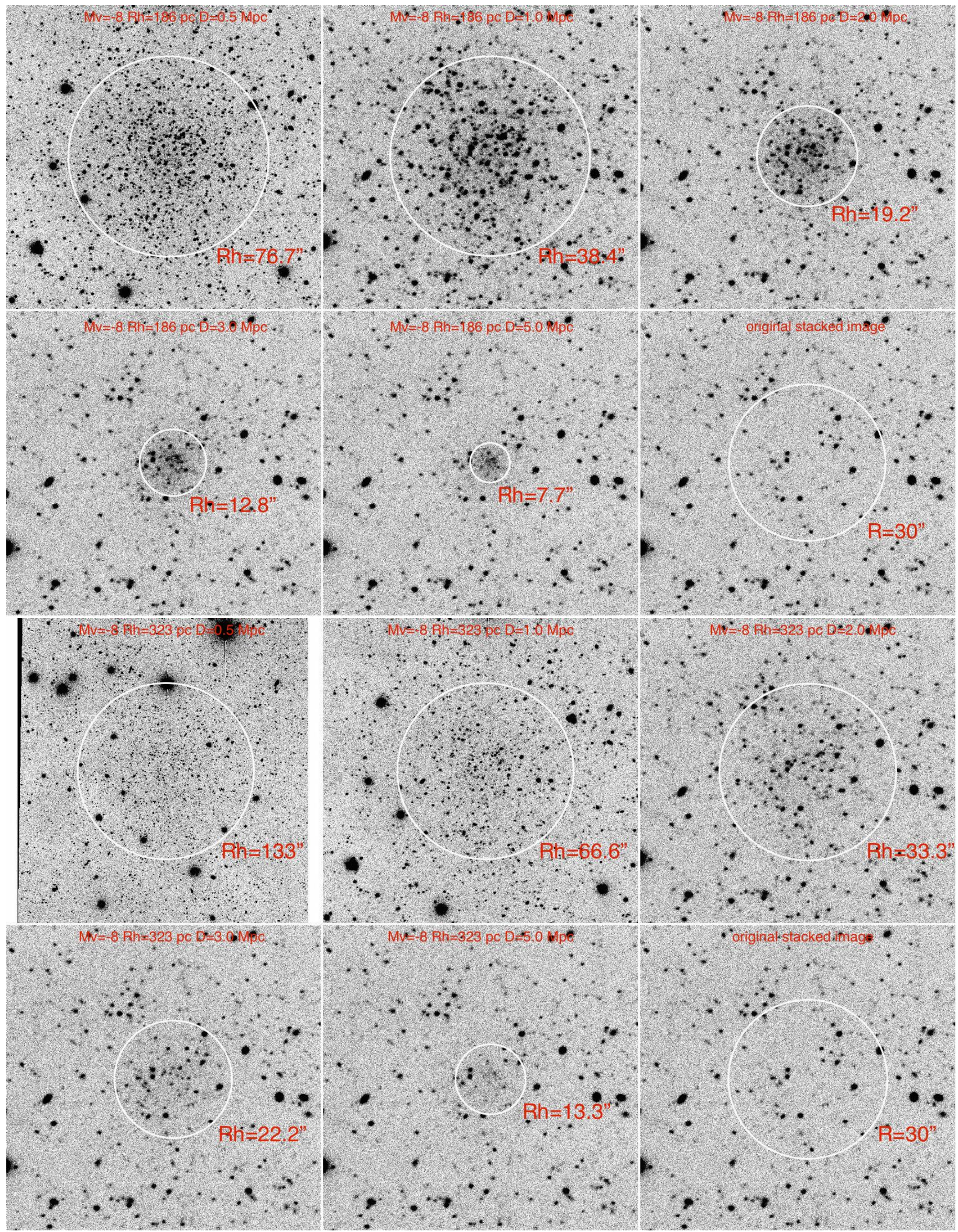

Fig. B.4. Best case observations, $M_{V}=-8.0$, compact (upper series of panels) and average models (lower series of panels). 


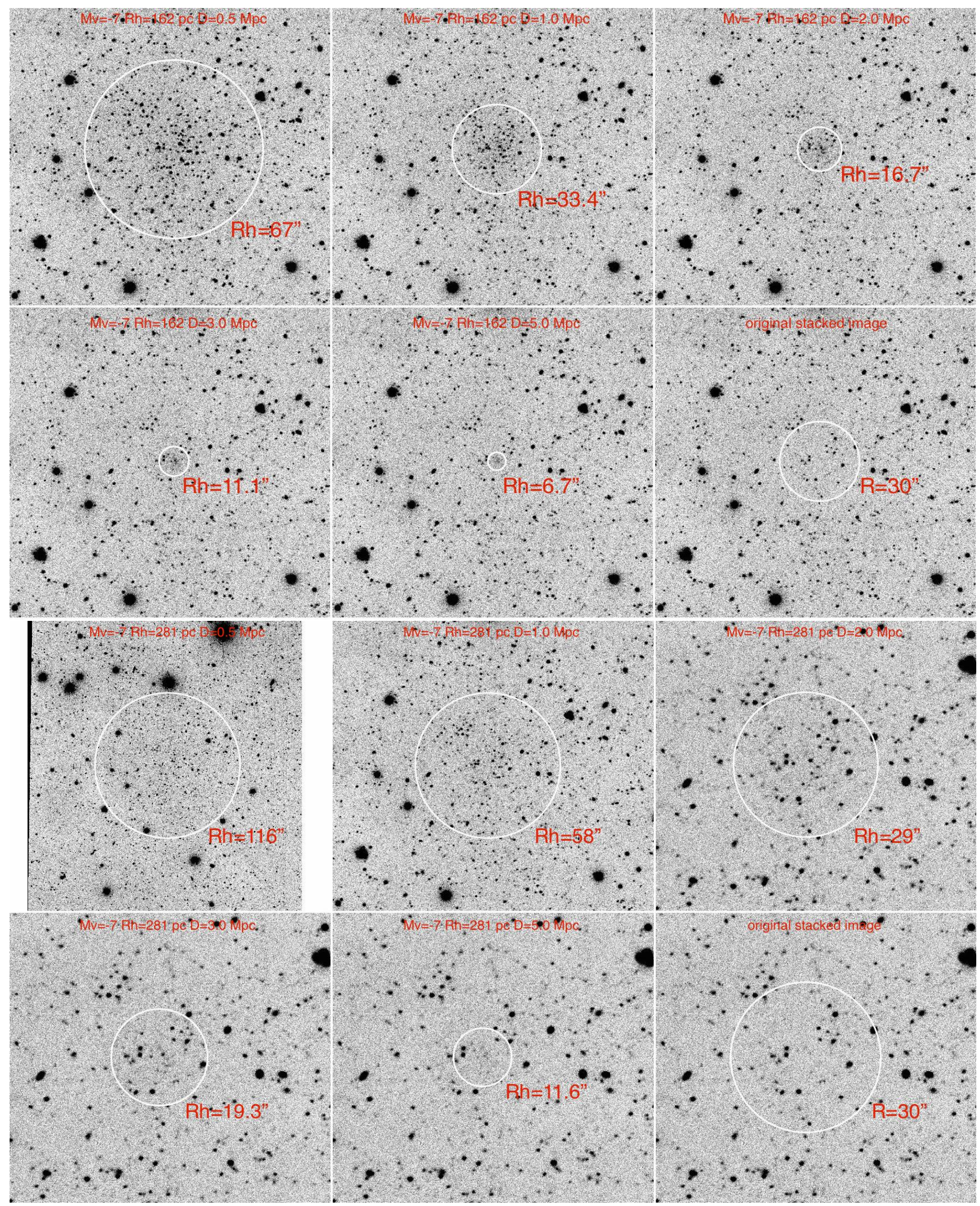

Fig. B.5. Best case observations, $M_{V}=-7.0$, compact (upper series of panels) and average models (lower series of panels). 